\title{
DECOMPOSING SYMMETRIC POWERS OF CERTAIN MODULAR REPRESENTATIONS OF CYCLIC GROUPS
}

\author{
R. JAMES SHANK AND DAVID L. WEHLAU
}

\begin{abstract}
For a prime number $p$, we construct a generating set for the ring of invariants for the $p+1$ dimensional indecomposable modular representation of a cyclic group of order $p^{2}$, and show that the Noether number for the representation is $p^{2}+p-3$. We then use the constructed invariants to explicitly describe the decomposition of the symmetric algebra as a module over the group ring, confirming the Periodicity Conjecture of Ian Hughes and Gregor Kemper for this case. In the appendix, we use our results to compute the Hilbert series for the corresponding ring of invariants together with some other related generating functions.
\end{abstract}

This paper is dedicated to Gerry Schwarz, on the occasion of his sixtieth birthday.

\section{INTRODUCTION}

Suppose that $V$ is a finite dimensional representation of a finite group $G$ over a field $\mathbf{F}$, i.e., $V$ is a finitely generated module over the group ring $\mathbf{F} G$. The action of $G$ on $V$ induces an action on the dual $V^{*}$ which extends to an action by algebra automorphisms on the symmetric algebra $\mathbf{F}[V]:=S\left(V^{*}\right)$. The elements of $V^{*}$, and thus also the elements of $\mathbf{F}[V]$, represent $\mathbf{F}$-valued functions on $V$. If $\left\{x_{1}, x_{2}, \ldots, x_{n}\right\}$ is a basis for $V^{*}$ then $\mathbf{F}[V]$ can be identified with the ring of polynomials $\mathbf{F}\left[x_{1}, x_{2}, \ldots, x_{n}\right]$. Let $\mathbf{F}[V]_{d}$ denote the subspace of homogeneous polynomials of degree $d$. Since the action of $G$ preserves degree, $\mathbf{F}[V]_{d}$ is a module over $\mathbf{F} G$ and

$$
\mathbf{F}[V]=\bigoplus_{d=0}^{\infty} \mathbf{F}[V]_{d}
$$

is a decomposition into a direct sum of finite dimensional $\mathbf{F} G$-modules. Of course $\mathbf{F}[V]_{d}$ is precisely the the $d^{\text {th }}$ symmetric power of $V^{*}$. Understanding the action of $G$ on $\mathbf{F}[V]_{d}$, and hence the action on $\mathbf{F}[V]$, is an important problem in representation theory. The primary goal is to write $\mathbf{F}[V]_{d}$ as a direct sum of indecomposable $\mathbf{F} G$-modules, refining the given decomposition of $\mathbf{F}[V]$. This means decomposing $\mathbf{F}[V]_{d}$ for infinitely many $d$. An important aspect of the group action is the ring

Date: February 2, 2008.

1991 Mathematics Subject Classification. 13A50.

The research of the first author is supported by grants from EPSRC.

The research of the second author is supported by grants from ARP and NSERC. 
of invariants

$$
\mathbf{F}[V]^{G}:=\{f \in \mathbf{F}[V] \mid g(f)=f, \forall g \in G\}
$$

a finitely generated subalgebra of $\mathbf{F}[V]$. A fundamental problem in invariant theory is the construction of a finite generating set for $\mathbf{F}[V]^{G}$. Since $G$ is finite, $\mathbf{F}[V]$ is a finite module over $\mathbf{F}[V]^{G}$. Thus $\mathbf{F}[V]$ is a module over both $\mathbf{F}[V]^{G}$ and $\mathbf{F} G$. Perhaps the right approach is to study $\mathbf{F}[V]$ as a finitely generated module over the extended group ring $\mathbf{F}[V]^{G} G$. Certainly, in the work of both Karagueuzian \& Symonds [11] and Hughes \& Kemper [10], the finite $\mathbf{F}[V]^{G_{-}}$ module structure of $\mathbf{F}[V]$ has been used to reduce decomposing $\mathbf{F}[V]$ over $\mathbf{F} G$ to a finite problem.

For the remainder of the paper, we assume that $\mathbf{F}$ has characteristic $p$ for a prime number $p$, and that $G \cong \mathbf{Z} / p^{r}$ is a cyclic group of order $p^{r}$. Choose a generator $\sigma$ for $G$. The isomorphism type of a representation of $G$ is determined by the Jordan canonical form of $\sigma$. Since the order of $\sigma$ is a power of $p$, and since a field of characteristic $p$ has no non-trivial $p^{\text {th }}$ roots of unity, all the eigenvalues of $\sigma$ must be 1 . If $m \leq p^{r}$, then the $m \times m$ matrix over $\mathbf{F}$ consisting of a single Jordan block with eigenvalue 1 determines an indecomposable $\mathbf{F} G$-module which we denote by $V_{m}$. Note that if $m>p^{r}$, then the matrix has order greater than $p^{r}$ and does not determine a representation of $G$. It follows from the form of the matrix that $V_{m}$ is faithful if and only if $p^{r-1}<m \leq p^{r}$, and that $V_{m}$ is a cyclic $\mathbf{F} G$ module. It is clear that if the Jordan canonical form of $\sigma$ consists of more than one Jordan block then the representation will be decomposable. Thus the complete set of inequivalent indecomposable $\mathbf{F} G$-modules are, up to isomorphism, $V_{1}, V_{2}$, $\ldots, V_{p^{r}}$. Furthermore, from the Jordan canonical form it is easy to see that these modules are naturally embedded into one another: $V_{1} \subset V_{2} \subset V_{3} \subset \cdots \subset V_{p^{r}}$. Note that the one dimensional space of $G$-fixed points, $V_{m}^{G} \cong V_{1}$ is the socle of $V_{m}$. Moreover, $V_{1}$ is the unique irreducible module, $V_{p^{r}} \cong \mathbf{F} G$ is the unique projective indecomposable, and an $\mathbf{F G - m o d u l e ~ i s ~ p r o j e c t i v e ~ i f ~ a n d ~ o n l y ~ i f ~ i t ~ i s ~ i n j e c t i v e ~ ( s e e , ~}$ for example, [1, Ch. II]). Also, it is easy to see that the representation $V_{m}$ is induced from a representation of a proper subgroup of $G$ if and only if $p$ divides $m$.

For $f \in \mathbf{F}\left[V_{n}\right]$, we define the norm of $f$, denoted by $N^{G}(f)$, to be the product over the $G$-orbit of $f$. Clearly $N^{G}(f) \in \mathbf{F}\left[V_{n}\right]^{G}$. For a subgroup $L=\left\langle\sigma^{p^{t}}\right\rangle$, we define the relative transfer $\operatorname{Tr}_{L}^{G}:=\sum_{i=0}^{p^{t}-1} \sigma^{i} \in \mathbf{F} G$.

The two main results we prove in this article concern the representation $V_{p+1}$ and are stated as Theorem 1.2 and Theorem 1.3 below. The following example illustrates these theorems.

Example 1.1. Let $\mathbf{F}$ be any field of characteristic $p=3$. We consider the indecomposable four dimensional representation $V_{4}$ of the cyclic group $G=\mathbf{Z} / 9$ of order 9. The group $G$ contains the subgroup $L$ of order 3 . Theorem 1.2 asserts that $\mathbf{F}\left[V_{4}\right]^{G}$ is generated by $M=N^{G}\left(x_{3}\right)=x_{3}^{3}-x_{3} x_{2}^{2}+x_{3}^{2} x_{1}+x_{3} x_{2} x_{1}$, $N=N^{G}\left(x_{4}\right)=x_{4}^{9}-x_{4}^{3} x_{3}^{6}+\ldots$, and elements from the image of the relative transfer, $\operatorname{Tr}_{L}^{G}\left(\mathbf{F}\left[x_{4}^{3}-x_{4} x_{1}^{2}, x_{3}, x_{2}, x_{1}\right]\right)$. In fact, a Magma [3] computation shows that $\mathbf{F}\left[V_{4}\right]^{G}$ is minimally generated by $M$ and $N$ together with the following 9 
invariants:

$$
\begin{aligned}
\operatorname{Tr}_{L}^{G}\left(x_{3}\right) & =x_{1} \\
\operatorname{Tr}_{L}^{G}\left(-x_{3}^{2}\right) & =x_{2}^{2}+x_{1} x_{3}-x_{1} x_{2}-x_{1}^{2}, \\
\operatorname{Tr}_{L}^{G}\left(-x_{2} x_{3}^{2}\right) & =x_{2}^{3}-x_{1} x_{2}^{2}-x_{1}^{2} x_{3}+x_{1}^{3}, \\
\operatorname{Tr}_{L}^{G}\left(-x_{3}\left(x_{4}^{3}-x_{4} x_{1}^{2}\right)\right) & =x_{2} x_{3}^{3}+\ldots, \\
\operatorname{Tr}_{L}^{G}\left(x_{3}^{2}\left(x_{4}^{3}-x_{4} x_{1}^{2}\right)\right) & =x_{2} x_{3}^{4}+\ldots, \\
\operatorname{Tr}_{L}^{G}\left(x_{2} x_{3}^{2}\left(x_{4}^{3}-x_{4} x_{1}^{2}\right)\right) & =x_{2}^{2} x_{3}^{4}+\ldots, \\
\operatorname{Tr}_{L}^{G}\left(-x_{3}\left(x_{4}^{3}-x_{4} x_{1}^{2}\right)^{2}\right) & =x_{3}^{7}+\ldots, \\
\operatorname{Tr}_{L}^{G}\left(-x_{3}^{2}\left(x_{4}^{3}-x_{4} x_{1}^{2}\right)^{2}\right) & =x_{3}^{8}+\ldots, \\
\operatorname{Tr}_{L}^{G}\left(x_{2} x_{3}^{2}\left(x_{4}^{3}-x_{4} x_{1}^{2}\right)^{2}\right) & =x_{2} x_{3}^{8}+\ldots .
\end{aligned}
$$

\begin{tabular}{|c|c|c|c|c|c|c|c|c|}
\hline $\mathbf{F}[V]_{0}$ & $\cong$ & $V_{1}$, & & & & & & \\
\hline $\mathbf{F}[V]_{1}$ & $\cong$ & $V_{4}$, & & & & & & \\
\hline $\mathbf{F}[V]_{2}$ & $\cong$ & $V_{7}$ & $\oplus$ & $V_{3}$, & & & & \\
\hline $\mathbf{F}[V]_{3}$ & $\cong$ & $V_{2}$ & $\oplus$ & $V_{3}$ & $\oplus$ & $V_{6}$ & $\oplus$ & $V_{9}$, \\
\hline $\mathbf{F}[V]_{4}$ & $\cong$ & $V_{5}$ & $\oplus$ & $2 V_{3}$ & $\oplus$ & $V_{6}$ & $\oplus$ & $2 V_{9}$, \\
\hline $\mathbf{F}[V]_{5}$ & $\cong$ & $V_{8}$ & $\oplus$ & $3 V_{3}$ & $\oplus$ & $2 V_{6}$ & $\oplus$ & $3 V_{9}$ \\
\hline $\mathbf{F}[V]_{6}$ & $\cong$ & & & $4 V_{3}$ & $\oplus$ & $3 V_{6}$ & $\oplus$ & $6 V_{9}$, \\
\hline $\mathbf{F}[V]_{7}$ & $\cong$ & & & $5 V_{3}$ & $\oplus$ & $4 V_{6}$ & $\oplus$ & $9 V_{9}$, \\
\hline $\mathbf{F}[V]_{8}$ & $\cong$ & & & $6 V_{3}$ & $\oplus$ & $5 V_{6}$ & $\oplus$ & $13 V_{9}$ \\
\hline $\mathbf{F}[V]_{9}$ & $\cong$ & $V_{1}$ & $\oplus$ & $7 V_{3}$ & $\oplus$ & $6 V_{6}$ & $\oplus$ & $18 V_{9}$ \\
\hline $\mathbf{F}[V]_{10}$ & $\cong$ & $V_{4}$ & $\oplus$ & $8 V_{3}$ & $\oplus$ & $7 V_{6}$ & $\oplus$ & $24 V_{9}$ \\
\hline $\mathbf{F}[V]_{11}$ & $\cong$ & $V_{7}$ & $\oplus$ & $10 V_{3}$ & $\oplus$ & $8 V_{6}$ & $\oplus$ & $31 V_{9}$ \\
\hline $\mathbf{F}[V]_{12}$ & $\cong$ & $V_{2}$ & $\oplus$ & $11 V_{3}$ & $\oplus$ & $10 V_{6}$ & $\oplus$ & $40 V_{9}$, \\
\hline $\mathbf{F}[V]_{13}$ & $\cong$ & $V_{5}$ & $\oplus$ & $13 V_{3}$ & $\oplus$ & $11 V_{6}$ & $\oplus$ & $50 V_{9}$, \\
\hline $\mathbf{F}[V]_{14}$ & $\cong$ & $V_{8}$ & $\oplus$ & $15 V_{3}$ & $\oplus$ & $13 V_{6}$ & $\oplus$ & $61 V_{9}$, \\
\hline $\mathbf{F}[V]_{15}$ & $\cong$ & & & $17 V_{3}$ & $\oplus$ & $15 V_{6}$ & $\oplus$ & $75 V_{9}$ \\
\hline $\mathbf{F}[V]_{16}$ & $\cong$ & & & $19 V_{3}$ & $\oplus$ & $17 V_{6}$ & $\oplus$ & $90 V_{9}$, \\
\hline $\mathbf{F}[V]_{17}$ & $\cong$ & & & $21 V_{3}$ & $\oplus$ & $19 V_{6}$ & $\oplus$ & $107 V_{9}$ \\
\hline
\end{tabular}

The first few homogeneous components of $\mathbf{F}\left[V_{4}\right]$ decompose into indecomposable F $G$-modules as follows:

The (one dimensional) socle of the non-induced indecomposable summand in $\mathbf{F}\left[V_{4}\right]_{i}$ for $i=0,1,2, \ldots, 5$ may be chosen to contain $1, x_{1}, x_{1}^{2}, M, x_{1} M$ and $x_{1}^{2} M$ respectively.

For degrees $d \geq 9$, write $d=9 a+c$ where $0 \leq c \leq 8$. Then $\mathbf{F}[V]_{d} \cong \mathbf{F}[V]_{c} \oplus$ $\alpha V_{3} \oplus \beta V_{6} \oplus \gamma V_{9}$ for some non-negative integers $\alpha, \beta$ and $\gamma$. Furthermore if $0 \leq c \leq 5$ and if we denote by $f$ a non-zero element of the socle of the noninduced summand in $\mathbf{F}[V]_{c}$, then the non-induced summand in $\mathbf{F}[V]_{d}$ may be chosen such that its socle is spanned by $N^{a} f$.

In Section 2 we develop tools for decomposing $\mathbf{F}\left[V_{n}\right]$ as an $\mathbf{F} G$-module. We then specialise to $r=2$ and $n=p+1$. In Section [3] we construct generators for 
$\mathbf{F}\left[V_{p+1}\right]^{\mathbf{Z} / p^{2}}$. We apply the "ladder technique" described in [15, §7], using group cohomology and a spectral sequence argument, to prove the following.

Theorem 1.2. Suppose $G \cong \mathbf{Z} / p^{2}$ and let $L \cong \mathbf{Z} / p$ denote its non-trivial proper subgroup. The ring of invariants $\mathbf{F}\left[V_{p+1}\right]^{G}$ is generated by $N^{G}\left(x_{p}\right), N^{G}\left(x_{p+1}\right)$ and elements from the image of the relative transfer, $\operatorname{Tr}_{L}^{G}\left(\mathbf{F}\left[N^{L}\left(x_{p+1}\right), x_{p}, \ldots, x_{1}\right]\right)$.

Recall that the Noether number of a representation is the largest degree of an element in a minimal homogeneous generating set for the corresponding ring of invariants. In Section 4 we use the generating set given by Theorem 1.2 to show that, for $p>2$, the Noether number for $V_{p+1}$ is $p^{2}+p-3$. In Section 5 we use the constructed generating set to describe the $\mathbf{F Z} / p^{2}$-module structure of $\mathbf{F}\left[V_{p+1}\right]$, confirming the Periodicity Conjecture of Hughes \& Kemper [10, Conjecture 4.6] in this case and proving the following.

Theorem 1.3. Let $G \cong \mathbf{Z} / p^{2}$ and let $d$ be any non-negative integer. In the decomposition of $\mathbf{F}\left[V_{p+1}\right]_{d}$ into a direct sum of indecomposable $\mathbf{F} G$-modules there is at most one indecomposable summand $V_{m}$ which is not induced from a representation of a proper subgroup. In particular, writing $d=a p^{2}+b p+c$ where $0 \leq b, c<p$, there is exactly one non-induced indecomposable summand when $b \leq p-2$ and $\mathbf{F}\left[V_{p+1}\right]_{d}$ is an induced module when $b=p-1$. Moreover, if $b \leq p-2$ then the non-induced indecomposable summand is isomorphic to $V_{c p+b+1}$ and we may choose the decomposition of $\mathbf{F}\left[V_{p+1}\right]_{d}$ such that the socle of this summand, $V_{c p+b+1}^{G}$, is spanned by the invariant $N^{G}\left(x_{p+1}\right)^{a} N^{G}\left(x_{p}\right)^{b} x_{1}^{c}$.

We note that Symonds, in a recent paper [16] based on his joint work with Karagueuzian [11], has proven the Periodicity Conjecture of Hughes \& Kemper. He goes on to prove that for $p^{r-1}<n<p^{r}$ and $d<p^{r}$, the $\mathbf{F Z} / p^{r}$-module $\mathbf{F}\left[V_{n}\right]_{d}$ is isomorphic to $\Omega^{-d} \Lambda^{d}\left(V_{p^{r}-n}\right)$ modulo induced modules [16, Corollary 3.11]. Here $\Lambda^{d}$ denotes the $d^{\text {th }}$ exterior power and $\Omega^{-d}$ denotes the $d^{\text {th }}$ cokernel of a minimal injective resolution (see [2, page 30]). It is instructive to compare this with Theorem 1.3 and Example 1.1.

In the Appendix we compute the Hilbert series of $\mathbf{F}\left[V_{p+1}\right]^{\mathbf{Z} / p^{2}}$. We also compute generating functions encoding the number of summands of each isomorphism type in $\mathbf{F}\left[V_{p+1}\right]_{t}$.

\section{Preliminaries}

Let $G=\langle\sigma\rangle \cong \mathbf{Z} / p^{r}$. It will be convenient to define $\Delta:=\sigma-1 \in \mathbf{F} G$. It is easy to see that $\Delta$ acts as a twisted derivation on $\mathbf{F}\left[V_{n}\right]$, i.e., $\Delta(a \cdot b)=$ $a \Delta(b)+\Delta(a) \sigma(b)$. We denote the full transfer, $\operatorname{Tr}_{\langle 1\rangle}^{G}$, by $\operatorname{Tr}^{G}$ and the image of the relative transfer, $\operatorname{Tr}_{L}^{G}\left(\mathbf{F}\left[V_{n}\right]^{L}\right)$, by $\operatorname{Im} \operatorname{Tr}_{L}^{G}$. Clearly $\operatorname{Im} \operatorname{Tr}_{L}^{G}$ is an ideal in $\mathbf{F}\left[V_{n}\right]^{G}$. A simple calculation with binomial coefficients shows that $\Delta^{p^{t}}=\sigma^{p^{t}}-1$ and $\Delta^{p^{t}-1}=$ $\left(\sigma^{p^{t}}-1\right) /(\sigma-1)=\operatorname{Tr}_{L}^{G}$. We denote the group cohomology of $G$ with coefficients in the $\mathbf{F} G$-module $W$ by $H^{*}(G, W)$. Note that $H^{0}(G, W)$ is just the fixed subspace $W^{G}$. Furthermore, since $G$ is cyclic, $H^{2 i-1}(G, W)=\operatorname{ker}\left(\left.\operatorname{Tr}^{G}\right|_{W}\right) / \operatorname{Im}\left(\left.\Delta\right|_{W}\right)$ and 
$H^{2 i}(G, W)=\operatorname{ker}\left(\left.\Delta\right|_{W}\right) / \operatorname{Im}\left(\left.\operatorname{Tr}^{G}\right|_{W}\right)$ for $i>0$ (see, for example, [6, §2.1]). It is clear from the definition of group cohomology that $H^{i}(G, P)=0$ if $i>0$ and $P$ is projective. Thus $H^{1}\left(G, V_{p^{r}}\right)=H^{2}\left(G, V_{p^{r}}\right)=0$. Furthermore, if $V_{m}$ is generated as an $\mathbf{F} G$-module by $e$, then $H^{0}\left(G, V_{m}\right)=V_{m}^{G}=\operatorname{span}_{\mathbf{F}}\left(\Delta^{m-1}(e)\right)$ and $\left\{e, \Delta(e), \ldots, \Delta^{m-1}(e)\right\}$ is a vector space basis for $V_{m}$. If we identify $V_{m-1}$ with the submodule $\Delta\left(V_{m}\right)$, then, for $m<p^{r}, H^{1}\left(G, V_{m}\right)$ is the one dimensional vector space $V_{m} / V_{m-1}$ and $H^{2}\left(G, V_{m}\right)$ is the one dimensional vector space $V_{m}^{G}$.

Let $W$ be any finite dimensional $\mathbf{F} G$-module. Define $\mathcal{L}_{t}(W):=\Delta^{t-1}(W)$. Clearly $\mathcal{L}_{i+1}(W) \subseteq \mathcal{L}_{i}(W)$. Furthermore, since $\sigma$ has order $p^{r}, \mathcal{L}_{p^{r}+1}(W)=0$. Thus we have the following filtration of $W$ by $\mathbf{F} G$-modules:

$$
W=\mathcal{L}_{1}(W) \supseteq \mathcal{L}_{2}(W) \supseteq \mathcal{L}_{3}(W) \supseteq \cdots \supseteq \mathcal{L}_{p^{r}}(W) \supseteq \mathcal{L}_{p^{r}+1}(W)=0 .
$$

This filtration of $W$ obviously induces a filtration of the subspace $W^{G}$ :

$$
W^{G}=\mathcal{L}_{1}^{G}(W) \supseteq \mathcal{L}_{2}^{G}(W) \supseteq \mathcal{L}_{3}^{G}(W) \supseteq \cdots \supseteq \mathcal{L}_{p^{r}}^{G}(W) \supseteq \mathcal{L}_{p^{r}+1}^{G}(W)=0
$$

where $\mathcal{L}_{t}^{G}(W):=\mathcal{L}_{t}(W) \cap W^{G}$.

Definition 2.1. For a non-zero $f \in W$, we define the length of $f$, denoted by $\ell(f)$, by $\ell(f) \geq t \Longleftrightarrow f \in \mathcal{L}_{t}(W)$. Note that $1 \leq \ell(f) \leq p^{r}$. We will refer to the above filtration of $W$ as the length filtration and say that a basis $\mathcal{B}$ for $W^{G}$ is compatible with the length filtration if $\mathcal{L}_{t}^{G}(W) \cap \mathcal{B}$ is a basis for $\mathcal{L}_{t}^{G}(W)$ for all $t$ (using the convention that the empty set is a basis for the zero vector space).

Lemma 2.2. If $W$ is a finite dimensional $\mathbf{F} G$-module, then

$$
\operatorname{dim}(W)=\sum_{t=1}^{p^{r}} t\left(\operatorname{dim}\left(\mathcal{L}_{t}^{G}(W)\right)-\operatorname{dim}\left(\mathcal{L}_{t+1}^{G}(W)\right)\right.
$$

Proof. Choose a decomposition of $W$ into indecomposable $\mathbf{F} G$-modules. For each indecomposable summand, choose a basis in which $\sigma$ is in Jordan canonical form. The union of these bases gives a basis for $W$. Intersecting this basis with $W^{G}$ gives a basis for $W^{G}$, say $\mathcal{B}$, which is compatible with the length filtration. It is clear that the number of elements in $\mathcal{B} \cap\left(\mathcal{L}_{t}^{G}(W) \backslash \mathcal{L}_{t+1}^{G}(W)\right)$ coincides with the number of indecomposable modules in the decomposition which are isomorphic to $V_{t}$, giving the required formula.

Suppose that $W$ is a finite dimensional $\mathbf{F} G$-module and $\mathcal{B}$ is a basis for $W^{G}$ which is compatible with the length filtration. For each $\alpha \in \mathcal{B}$ choose $\gamma \in W$ with $\Delta^{\ell(\alpha)-1}(\gamma)=\alpha$. (The existence of a suitable $\gamma$ follows from the definition of length.) Define $V(\alpha)$ to be the $\mathbf{F} G$-module generated by $\gamma$. Note that $\alpha$ spans the socle of $V(\alpha)$ and that $\operatorname{dim}(V(\alpha))=\ell(\alpha)$.

\section{Proposition 2.3.}

$$
W=\bigoplus_{\alpha \in \mathcal{B}} V(\alpha)
$$

Proof. The natural homomorphism of the external direct sum of the $V(\alpha)$ to $W$ is injective on the socle and is therefore injective. Thus the internal sum of the 
$V(\alpha)$ is direct. It follows from Lemma 2.2 that the dimension of $W$ coincides with the dimension of $\oplus_{\alpha \in \mathcal{B}} V(\alpha)$, giving equality.

The above shows how we may obtain a direct sum decomposition of $W$ into indecomposable submodules from any basis of $W^{G}$ which is compatible with the length filtration of $W^{G}$. Clearly every such decomposition arises in this way. Furthermore, an element $f \in W^{G}$ has length $t$ if and only if there is an $\mathbf{F} G$ decomposition $W=W^{\prime} \oplus V_{t}$ with $f$ spanning $V_{t}^{G}$.

Note that if $f, h \in \mathbf{F}[V]^{G}$ then $\ell(f h) \geq \ell(f)$. To see this write $f=\Delta^{\ell(f)-1}(F)$. Then $f h=\Delta^{\ell(f)-1}(F h)$. In general it may happen that $\ell(f h)>\max \{\ell(f), \ell(h)\}$. Computer computations together with various results, such as Proposition 5.3. lead us to make the following conjecture.

Conjecture 2.4. Suppose $f, h \in \mathbf{F}[V]^{G}$ with $\ell(f) \equiv 0(\bmod p)$. Then $\ell(f h) \equiv 0$ $(\bmod p)$.

For $n \leq p^{r}$, choose an $\mathbf{F} G$-module generator $x_{n}$ for $V_{n}^{*}$ and define $x_{i}=\Delta^{n-i}\left(x_{n}\right)$ for $i=1, \ldots, n-1$. Then $\left\{x_{1}, x_{2}, \ldots, x_{n}\right\}$ is a basis of $V_{n}^{*}$. Let $\overline{\mathbf{F}}$ denote the algebraic closure of $\mathbf{F}$ and define $\overline{V_{n}}:=\overline{\mathbf{F}} \otimes_{\mathbf{F}} V_{n}$. Let $\left\{e_{1}, e_{2}, e_{3}, \ldots, e_{n}\right\}$ denote the basis for $\overline{V_{n}}$ dual to $\left\{1 \otimes x_{1}, \ldots, 1 \otimes x_{n}\right\}$. Note that $e_{1}$ generates $\overline{V_{n}}$ as an $\overline{\mathbf{F}} G$-module and that $\Delta\left(e_{n}\right)=0$. Using the inclusion $\mathbf{F} \subseteq \overline{\mathbf{F}}$, allows us to interpret elements of $\mathbf{F}\left[V_{n}\right]$ as regular functions on $\overline{V_{n}}$, i.e., we identify $\mathbf{F}\left[V_{n}\right]$ in a natural way with a subset of $\overline{\mathbf{F}}\left[\overline{V_{n}}\right]$. For a subset $X \subseteq \overline{\mathbf{F}}\left[\overline{V_{n}}\right]$, define $\mathcal{V}(X)=\left\{v \in \overline{V_{n}} \mid\right.$ $f(v)=0 \forall f \in X\}$.

Lemma 2.5. Suppose $p^{r-1}<n \leq p^{r}$ and let $H$ denote the subgroup $\left\langle\sigma^{p^{t+1}}\right\rangle \cong$ $\mathbf{Z} / p^{r-t-1}$ of $G=\langle\sigma\rangle \cong \mathbf{Z} / p^{r}$ where $0 \leq t \leq r-1$.

(1) $\mathcal{V}\left(\operatorname{Im} \operatorname{Tr}_{H}^{G}\right)={\overline{V_{n}}}^{\mathbf{Z} / p^{r-t}}=\operatorname{span}_{\overline{\mathbf{F}}}\left\{e_{n-p^{t}+1}, e_{n-p^{t}+2}, \ldots, e_{n-1}, e_{n}\right\}$.

(2) For $f \in \mathbf{F}\left[V_{n}\right]^{G}$, if $\ell(f) \geq p^{t}+1$ then

$$
f \in \sqrt{\operatorname{Im} \operatorname{Tr}_{H}^{G}}=\left(\left(x_{1}, x_{2}, \ldots, x_{n-p^{t}}\right) \mathbf{F}\left[V_{n}\right]\right) \cap \mathbf{F}\left[V_{n}\right]^{G} .
$$

Proof. The equality $V_{n}^{\mathbf{Z} / p^{r-t}}=\operatorname{span}_{\mathbf{F}}\left\{e_{n-p^{t}+1}, e_{n-p^{t}+2}, \ldots, e_{n-1}, e_{n}\right\}$ is easily verified. The equality $\mathcal{V}\left(\operatorname{Im} \operatorname{Tr}_{H}^{G}\right)={\overline{V_{n}}}^{\mathbf{Z} / p^{r-t}}$ follows from [8, Proposition 12.5] (see also [5, Theorem 12]). This equality of sets may be expressed equivalently as the equality of ideals $\sqrt{{\operatorname{Im~} \operatorname{Tr}_{H}^{G}}^{\prime}}=\left(\left(x_{1}, x_{2}, \ldots, x_{n-p^{t}}\right) \mathbf{F}\left[V_{n}\right]\right) \cap \mathbf{F}\left[V_{n}\right]^{G}$ (see, for example, [5, Proposition 11]). Thus it only remains to show that if $\ell(f) \geq p^{t}+1$ then $f \in \sqrt{\operatorname{Im} \operatorname{Tr}_{H}^{G}}$.

To see this suppose that $\ell(f) \geq p^{t}+1$. Then $f=\Delta^{p^{t}}(F)$ for some $F \in$ $\mathbf{F}\left[V_{n}\right]$. Therefore $f\left(e_{i}\right)=\left(\Delta^{p^{t}} F\right)\left(e_{i}\right)=\left((\sigma-1)^{p^{t}} F\right)\left(e_{i}\right)=\left(\sigma^{p^{t}}(F)-F\right)\left(e_{i}\right)=$ $F\left(\sigma^{-p^{t}}\left(e_{i}\right)\right)-F\left(e_{i}\right)$. Thus $f\left(e_{i}\right)=0$ if $e_{i}$ is fixed by $\sigma^{p^{t}}$, i.e., if $i \geq n-p^{t}+1$. Therefore if $\ell(f) \geq p^{t}+1$ then $f$ vanishes on the set $\operatorname{span}_{\mathbf{F}}\left\{e_{n-p^{t}+1}, e_{n-p^{t}+2}, \ldots, e_{n-1}, e_{n}\right\}$. Hence if $\ell(f) \geq p^{t}+1$ then $f \in \sqrt{\operatorname{Im} \operatorname{Tr}_{H}^{G}}$ 
Proposition 2.6. Suppose that $f$ is a non-zero homogeneous element of $\mathbf{F}\left[V_{n}\right]^{G}$. Then $\ell\left(f N^{G}\left(x_{n}\right)\right)=\ell(f)$.

Proof. Denote $N^{G}\left(x_{n}\right)$ by $N$. Define $t$ such that $p^{t-1}<n \leq p^{t}$ (the case $n=1$ is trivial). Then the leading term of $N$ is $x_{n}^{p^{t}}$. Let $\mathbf{F}\left[V_{n}\right]^{b}$ denote the span of the monomials in $\mathbf{F}\left[V_{n}\right]$ which, as polynomials in $x_{n}$, have degree less than $p^{t}$. The fact that $x_{n} \notin \Delta\left(\mathbf{F}\left[V_{n}\right]\right)$ means that $\mathbf{F}\left[V_{n}\right]^{b}$ is a $\mathbf{F} G$-submodule of $\mathbf{F}\left[V_{n}\right]$. For an arbitrary polynomial $h \in \mathbf{F}\left[V_{n}\right]$, viewing $h$ as a polynomial in $x_{n}$ and dividing by $N^{G}\left(x_{n}\right)$ gives $h=q N+r$ for unique $r \in \mathbf{F}\left[V_{n}\right]^{b}$ and $q \in \mathbf{F}\left[V_{n}\right]$. This gives the $\mathbf{F} G$ module decomposition $\mathbf{F}\left[V_{n}\right]=N \mathbf{F}\left[V_{n}\right] \oplus \mathbf{F}\left[V_{n}\right]^{b}$ (compare with [10, Lemma 2.9] and [14, $\S 2])$. As noted above $\ell(N f) \geq \ell(f)$. Suppose $N f=\Delta^{t}(F)$ and write $F=N F_{1}+F_{0}$ with $F_{0} \in \mathbf{F}\left[V_{n}\right]^{b}$. Then $N f=\Delta^{t}\left(N F_{1}+F_{0}\right)=N \Delta^{t}\left(F_{1}\right)+\Delta^{t}\left(F_{0}\right)=$ $N \Delta^{t}\left(F_{1}\right)$ and thus $f=\Delta^{t}\left(F_{1}\right)$. This shows that $\ell(f) \geq \ell(N f)$.

\section{Computing $\mathbf{F}\left[V_{p+1}\right]^{\mathbf{z} / p^{2}}$}

In this section we use the ladder technique described in [15, $§ 7]$ to prove Theorem 1.2. We use the notation

$$
G:=\langle\sigma\rangle \cong \mathbf{Z} / p^{2}, L:=\left\langle\sigma^{p}\right\rangle \cong \mathbf{Z} / p \text {, and } Q:=G / L=\langle\bar{\sigma}\rangle \cong \mathbf{Z} / p .
$$

Note that $\operatorname{deg}\left(N^{G}\left(x_{p}\right)\right)=p, \operatorname{deg}\left(N^{G}\left(x_{p+1}\right)\right)=p^{2}$ and $\operatorname{Tr}_{L}^{G}\left(x_{p}\right)=x_{1}$.

The action of $L$ on $V_{p+1}^{*}$ is given by $\sigma^{p}\left(x_{p+1}\right)=x_{p+1}+x_{1}$ and $\sigma^{p}\left(x_{i}\right)=x_{i}$ for $i \leq p$. Thus as $L$-modules, $\mathbf{F}\left[V_{p+1}\right] \cong \mathbf{F}\left[V_{2} \oplus(p-1) V_{1}\right]$. Therefore $\mathbf{F}\left[V_{p+1}\right]^{L} \cong$ $\mathbf{F}\left[N^{L}\left(x_{p+1}\right), x_{p}, \ldots, x_{1}\right]$ with $N^{L}\left(x_{p+1}\right)=x_{p+1}^{p}-x_{1}^{p-1} x_{p+1}$. The action of $Q$ on $\mathbf{F}\left[V_{p+1}\right]^{L}$ is given by $\bar{\sigma}\left(N^{L}\left(x_{p+1}\right)\right)=N^{L}\left(x_{p+1}\right)+x_{p}^{p}-x_{1}^{p-1} x_{p}$ and $\bar{\sigma}\left(x_{i}\right)=\sigma\left(x_{i}\right)$ for $i=1,2, \ldots, p$. Define

$$
A:=\mathbf{F}\left[z_{p}, \ldots, z_{1}, X_{p}, \ldots, X_{1}\right]
$$

with $\operatorname{deg}\left(z_{i}\right)=p$ and $\operatorname{deg}\left(X_{i}\right)=1$. Further define an algebra homomorphism $\pi: A \rightarrow \mathbf{F}\left[V_{p+1}\right]^{L}$ by $\pi\left(z_{i}\right)=x_{i+1}^{p}-x_{1}^{p-1} x_{i+1}$ and $\pi\left(X_{i}\right)=x_{i}$. Note that $\pi$ is a degree preserving surjection with $\pi\left(z_{p}\right)=N^{L}\left(x_{p+1}\right)$. Further note that the kernel of $\pi$ is the ideal

$$
I:=\left(z_{p-1}-\left(X_{p}^{p}-X_{1}^{p-1} X_{p}\right), \ldots, z_{1}-\left(X_{2}^{p}-X_{1}^{p-1} X_{2}\right)\right) A .
$$

Define an action, by algebra automorphisms, of $Q$ on $A$ by taking $\bar{\sigma}\left(z_{i}\right)=z_{i}+z_{i-1}$ and $\bar{\sigma}\left(X_{i}\right)=X_{i}+X_{i-1}$ for $i>1, \bar{\sigma}\left(z_{1}\right)=z_{1}$ and $\bar{\sigma}\left(X_{1}\right)=X_{1}$. Thus as $\mathbf{F} Q_{-}$ modules $A \cong \mathbf{F}\left[2 V_{p}\right]$ and $\pi$ is a map of $\mathbf{F} Q$-modules.

The short exact sequence of $\mathbf{F} Q$-modules, $0 \rightarrow I \rightarrow A \stackrel{\pi}{\longrightarrow} \mathbf{F}\left[V_{p+1}\right]^{L} \rightarrow 0$, gives a long exact sequence on group cohomology

$$
0 \rightarrow I^{Q} \rightarrow A^{Q} \rightarrow\left(\mathbf{F}\left[V_{p+1}\right]^{L}\right)^{Q} \rightarrow H^{1}(Q, I) \rightarrow H^{1}(Q, A) \rightarrow \cdots
$$

We will show that the inclusion of $I$ into $A$ induces an injection of $H^{1}(Q, I)$ into $H^{1}(Q, A)$. Thus $\pi$ restricts to a surjection from $A^{Q}$ to $\left(\mathbf{F}\left[V_{p+1}\right]^{L}\right)^{Q}=\mathbf{F}\left[V_{p+1}\right]^{G}$. Since $2 V_{p}$ is a permutation representation of $Q$, after a suitable change of basis, $Q$ acts on $A$ by permuting the variables. Using the permutation basis, the orbit 
sums of monomials form a vector space basis for $A^{Q}$. Since $Q \cong \mathbf{Z} / p$, these orbits are of size $p$ or size 1 . The orbits of size $p$ span projective $\mathbf{F} Q$-module summands of $\mathbf{F}\left[V_{p+1}\right]^{L}$ while the orbits of size 1 span trivial summands. It easy to see that, in the original basis, the orbits of size 1 are polynomials in $N^{Q}\left(z_{p}\right)$ and $N^{Q}\left(X_{p}\right)$, while the orbit sums coming from orbits of size $p$ are elements in the image of the transfer. Thus $A^{Q}$ is generated by $N^{Q}\left(z_{p}\right), N^{Q}\left(X_{p}\right)$ and elements from $\operatorname{Tr}^{Q}(A)$, giving Theorem 1.2 .

The rest of this section is devoted to completing the proof of Theorem 1.2 by showing that the inclusion of $I$ into $A$ induces an injection of $H^{1}(Q, I)$ into $H^{1}(Q, A)$ (see Theorem $\left.3.10(\mathrm{~b})\right)$. We start by describing $H^{*}(Q, A)$.

Proposition 3.1. (a) $H^{2}(Q, A)=A^{Q} / \operatorname{Tr}^{Q}(A) \cong \mathbf{F}\left[N^{Q}\left(X_{p}\right), N^{Q}\left(z_{p}\right)\right]$.

(b) $H^{1}(Q, A)$ is a principal $A^{Q}$-module with annihilator given by $\operatorname{Tr}^{Q}(A)$.

Proof. It follows from the discussion above that, as an F $Q$-module, $A$ consists of projective summands and trivial summands with the trivial summands spanned by the monomials in $N^{Q}\left(X_{p}\right)$ and $N^{Q}\left(z_{p}\right)$. The projective summands do not contribute to the cohomology. The trivial summands contribute non-zero classes to both the first and second cohomology.

Note that, although $H^{1}(Q, A)$ does not have a multiplicative structure, it is isomorphic to $\mathbf{F}\left[N^{Q}\left(X_{p}\right), N^{Q}\left(z_{p}\right)\right]$ as an $A^{Q}$-module.

To compute $H^{*}(Q, I)$, we start by resolving $I$ as an $A-\mathbf{F} Q$-module using a Koszul resolution (see, for example, [4, §1.6]). Observe that $I$ is generated by an $A$-regular sequence of length $p-1$. Furthermore, these generators span the degree $p$ homogeneous component, $I_{p}$, of $I$ and, as a $\mathbf{F} Q$-module, $I_{p} \cong V_{p-1}$. Let $\mu$ denote the $Q$-equivariant map from $V_{p-1} \otimes A$ to $A$ given by identifying elements of $V_{p-1}$ with elements of $I_{p}$ and then using the multiplication in $A$. Let $\Lambda^{i}\left(V_{p-1}\right)$ denote the $i^{\text {th }}$ exterior power of $V_{p-1}$. Define $\zeta^{i}: \Lambda^{i}\left(V_{p-1}\right) \rightarrow \Lambda^{i-1}\left(V_{p-1}\right) \otimes V_{p-1}$ by

$$
\zeta^{i}\left(v_{1} \wedge v_{2} \wedge \cdots \wedge v_{i}\right)=\sum_{j=1}^{i}(-1)^{i-j}\left(v_{1} \wedge \cdots \wedge \widehat{v_{j}} \wedge \cdots \wedge v_{i}\right) \otimes v_{j}
$$

for all $v_{1}, v_{2}, \ldots, v_{i} \in V_{p-1}$. Define $F^{-i}:=\Lambda^{i}\left(V_{p-1}\right) \otimes A$ for $i=1,2, \ldots, p-1$ and define $\rho^{-i}: F^{-i} \rightarrow F^{-i+1}$ to be $\left(1_{\Lambda^{i-1}\left(V_{p-1}\right)} \otimes \mu\right) \circ\left(\zeta^{i} \otimes 1_{A}\right)$. This gives the following sequence of $A-\mathbf{F} Q$-modules:

$$
0 \rightarrow F^{1-p} \stackrel{\rho^{1-p}}{\longrightarrow} F^{2-p} \stackrel{\rho^{2-p}}{\longrightarrow} \cdots \stackrel{\rho^{-3}}{\longrightarrow} F^{-2} \stackrel{\rho^{-2}}{\longrightarrow} F^{-1} \stackrel{\mu}{\longrightarrow} I \rightarrow 0 .
$$

Since the generators of $I$ form a regular $A$-sequence, it follows from [4, Corollary 1.6.14] that this sequence is exact. For $i>0$, define $K^{-i}$ to be the kernel of the map $\rho^{-i}: F^{-i} \rightarrow F^{-i+1}$. For convenience, we define $K^{0}:=I, K^{1}:=A / I$, $K^{a}:=0$ for $a>1, F^{1}:=A / I$ and $F^{a}:=0$ for $a>1$. Using the exactness of the resolution, we get a series of short exact sequences $0 \rightarrow K^{-i} \rightarrow F^{-i} \rightarrow K^{-i+1} \rightarrow 0$. For each of these short exact sequences, we apply group cohomology to get a long 
exact sequence:

$$
\begin{aligned}
0 \rightarrow H^{0}\left(Q, K^{-i}\right) \rightarrow H^{0}\left(Q, F^{-i}\right) & \rightarrow H^{0}\left(Q, K^{-i+1}\right) \rightarrow H^{1}\left(Q, K^{-i}\right) \\
& \rightarrow H^{1}\left(Q, F^{-i}\right) \rightarrow H^{1}\left(Q, K^{-i+1}\right) \rightarrow \ldots
\end{aligned}
$$

Defining $D^{a, b}:=H^{b}\left(Q, K^{a}\right)$ and $E^{a, b}:=H^{b}\left(Q, F^{a}\right)$ gives a bigraded exact couple which leads to a spectral sequence. This is essentially the construction given at the end of [12, Ch XI, §5]. We will use this spectral sequence to describe $H^{1}(Q, I)$. The following series of lemmas lead to a description of $H^{b}\left(Q, F^{a}\right)$.

Lemma 3.2. The map $\zeta^{i+1}$ is an isomorphism of $\mathbf{F} Q$-modules from $\Lambda^{i+1}\left(V_{p-1}\right)$ to a direct summand of $\Lambda^{i}\left(V_{p-1}\right) \otimes V_{p-1}$.

Proof. It is clear that $\zeta^{i+1}$ followed by the natural projection from $\Lambda^{i}\left(V_{p-1}\right) \otimes V_{p-1}$ to $\Lambda^{i+1}\left(V_{p-1}\right)$ is $i+1$ times the identity map on $\Lambda^{i+1}\left(V_{p-1}\right)$. Since $i+1 \leq p-1$, this is an isomorphism.

Lemma 3.3. $V_{p-1} \otimes V_{p-1} \cong V_{1} \oplus(p-2) V_{p}$.

Proof. If the representation $\operatorname{ring} R_{\mathbf{F Z} / p}$ is extended by adjoining an element $\alpha$ satisfying $V_{2}=\alpha+\alpha^{-1}$, then by [10, Lemma 2.3], $V_{n}=\frac{\alpha^{n}-\alpha^{-n}}{\alpha-\alpha^{-1}}$. Thus, in the augmented representation ring,

$$
\begin{aligned}
V_{p-1}^{2}-V_{p} \cdot V_{p-2} & =\frac{\left.\left(\alpha^{p-1}-\alpha^{-(p-1)}\right)^{2}-\left(\alpha^{p}-\alpha^{-p}\right)\left(\alpha^{p-2}-\alpha^{-(p-2)}\right)\right)}{\left(\alpha-\alpha^{-1}\right)^{2}} \\
& =\frac{\alpha^{2 p-2}-2+\alpha^{-(2 p-2)}-\left(\alpha^{2 p-2}-\alpha^{2}-\alpha^{-2}+\alpha^{-(2 p-2)}\right)}{\alpha^{2}-2+\alpha^{-2}} \\
& =1=V_{1} .
\end{aligned}
$$

Therefore, $V_{p-1} \otimes V_{p-1} \cong V_{p} \otimes V_{p-2} \oplus V_{1}$. It follows from [1, Ch. II $\S 7$ Lemma 4] that $V_{p} \otimes V_{i} \cong i V_{p}$. Thus $V_{p-1} \otimes V_{p-1} \cong(p-2) V_{p} \oplus V_{1}$, as required.

Lemma 3.4. For $i$ even,

$$
\Lambda^{i}\left(V_{p-1}\right) \cong V_{1} \oplus \frac{1}{p}\left(\left(\begin{array}{c}
p-1 \\
i
\end{array}\right)-1\right) V_{p}
$$

and for $i$ odd,

$$
\Lambda^{i}\left(V_{p-1}\right) \cong V_{p-1} \oplus \frac{1}{p}\left(\left(\begin{array}{c}
p-1 \\
i
\end{array}\right)-(p-1)\right) V_{p}
$$

Proof. First observe that $\operatorname{dim}_{\mathbf{F}}\left(\Lambda^{i}\left(V_{p-1}\right)=\left(\begin{array}{c}p-1 \\ i\end{array}\right) \equiv(-1)^{i}(\bmod p)\right.$. Therefore the dimensions are correct. Thus it follows from Lemma 3.2 that $\Lambda^{i}\left(V_{p-1}\right)$ is a non-projective summand of $\Lambda^{i-1}\left(V_{p-1}\right) \otimes V_{p-1}$. Also note that the result is true for $i=0$ and $i=1$. We proceed by induction. Suppose the result holds for $i$. For $i$ even this gives,

$$
\begin{aligned}
\Lambda^{i}\left(V_{p-1}\right) \otimes V_{p-1} & \left.\cong V_{1} \otimes V_{p-1} \oplus \frac{1}{p}\left(\left(\begin{array}{c}
p-1 \\
i
\end{array}\right)-1\right)\right) V_{p} \otimes V_{p-1} \\
& \left.\cong V_{p-1} \oplus \frac{p-1}{p}\left(\left(\begin{array}{c}
p-1 \\
i
\end{array}\right)-1\right)\right) V_{p}
\end{aligned}
$$


and therefore, $\Lambda^{i}\left(V_{p-1}\right) \otimes V_{p-1}$ is isomorphic to $V_{p-1}$ plus projective modules. Hence $\Lambda^{i+1}\left(V_{p-1}\right)$ is isomorphic to $V_{p-1}$ plus projective modules. For $i$ odd,

$$
\begin{aligned}
\Lambda^{i}\left(V_{p-1}\right) \otimes V_{p-1} & \cong V_{p-1} \otimes V_{p-1} \oplus \frac{1}{p}\left(\left(\begin{array}{c}
p-1 \\
i
\end{array}\right)-(p-1)\right) V_{p} \otimes V_{p-1} \\
& \cong V_{p-1} \otimes V_{p-1} \oplus \frac{p-1}{p}\left(\left(\begin{array}{c}
p-1 \\
i
\end{array}\right)-(p-1)\right) V_{p}
\end{aligned}
$$

and, therefore, $\Lambda^{i}\left(V_{p-1}\right) \otimes V_{p-1}$ is isomorphic to $V_{p-1} \otimes V_{p-1}$ plus projective modules. From Lemma 3.3, $V_{p-1} \otimes V_{p-1}$ is isomorphic to $V_{1}$ plus projective modules. Hence $\Lambda^{i+1}\left(V_{p-1}\right)$ is isomorphic to $V_{1}$ plus projective modules.

Lemma 3.5. For $a \leq 0$ and $b>0, H^{b}\left(Q, F^{a}\right)$ is a principal $A^{Q}$-module with annihilator $\operatorname{Tr}^{Q}(A)$.

Proof. As an $\mathbf{F} Q$-module, $A$ is a direct sum of projective summands with socles contained in $\operatorname{Tr}^{Q}(A)$ and one dimensional summands spanned by monomials in $N^{Q}\left(X_{p}\right)$ and $N^{Q}\left(z_{p}\right)$. It follows from Lemma 3.4 that $\Lambda^{-a}\left(V_{p-1}\right)$ contains a a single non-projective summand. Note that projective summands do not contribute to the cohomology. Further note that for any module $M$ and projective module $P$, $M \otimes P$ is projective. Thus $H^{b}\left(Q, \Lambda^{-a}\left(V_{p-1}\right)\right)$ is a one dimensional vector space and $H^{b}\left(Q, F^{a}\right) \cong H^{b}\left(Q, \Lambda^{-a}\left(V_{p-1}\right) \otimes A\right) \cong H^{b}(Q, A)$. The result follows from Proposition 3.1

The following lemma is a preliminary step in evaluating $d^{a, b}: E^{a, b} \rightarrow E^{a+1, b}$ for $a<0$ and $b>0$.

Lemma 3.6. The inclusion of $I_{p}$ into $A_{p}$ induces an injection from $H^{1}\left(Q, I_{p}\right)$ to $H^{1}\left(Q, A_{p}\right)$ and the zero map from $H^{2}\left(Q, I_{p}\right)$ to $H^{2}\left(Q, A_{p}\right)$.

Proof. To see that the inclusion induces an injection from $H^{1}\left(Q, I_{p}\right)$ to $H^{1}\left(Q, A_{p}\right)$, first note that $\Delta$ is a twisted derivation and that $\Delta(f)$, for $f$ a generator of $A$, lies in $\operatorname{Span}_{\mathbf{F}}\left(z_{p-1}, \ldots, z_{1}, X_{p-1}, \ldots, X_{1}\right)$. Therefore $\Delta(A)$ is contained in the ideal $\left(z_{p-1}, \ldots, z_{1}, X_{p-1}, \ldots, X_{1}\right) A$. As an $\mathbf{F} Q$-module, $I_{p}$ is isomorphic to $V_{p-1}$ with generator $r:=z_{p-1}-\left(X_{p}^{p}-X_{1}^{p-1} X_{p}\right)$. Thus $H^{1}\left(Q, I_{p}\right)$ is a one dimensional vector space with $r$ representing a non-zero cohomology class. Since $r$ does not lie in the ideal $\left(z_{p-1}, \ldots, z_{1}, X_{p-1}, \ldots, X_{1}\right) A$, this element does not lie in $\Delta(A)$ and, therefore, represents a non-zero class in $H^{1}\left(Q, A_{p}\right)$.

To see that inclusion induces the zero map from $H^{2}\left(Q, I_{p}\right)$ to $H^{2}\left(Q, A_{p}\right)$, observe that $I_{p} \cong V_{p-1}$ and $A_{p}$ is isomorphic to $V_{1}$ plus projectives. Thus the map on cohomology is determined by a $\mathbf{Z} / p$-equivariant map from $V_{p-1}$ to $V_{1}$, and all such maps induce the zero map in second cohomology.

For $a<0$, the first differential in the spectral sequence is the map on cohomology $d^{a, b}: H^{b}\left(Q, F^{a}\right) \rightarrow H^{b}\left(Q, F^{a+1}\right)$ induced by $\rho^{a}: F^{a} \rightarrow F^{a+1}$

Theorem 3.7. For $b>0$ and $a<0, d^{a, b}: H^{b}\left(Q, F^{a}\right) \rightarrow H^{b}\left(Q, F^{a+1}\right)$ is an isomorphism if $a$ and $b$ have the same parity and zero if $a$ and $b$ have different parities. 
Proof. From Lemma [3.5, $H^{b}\left(Q, F^{a}\right)$ is a principal $A^{Q}$-module. Since $d^{a, b}$ is an $A^{Q}$-module map, it is sufficient to evaluate $d^{a, b}$ on a generator. Thus, using the definition of $\rho^{a}$, we see that $d^{a, b}$ is determined by the composition

$$
\Lambda^{-a}\left(V_{p-1}\right) \stackrel{\zeta^{-a}}{\longrightarrow} \Lambda^{-a-1}\left(V_{p-1}\right) \otimes V_{p-1} \stackrel{\cong}{\longrightarrow} \Lambda^{-a-1}\left(V_{p-1}\right) \otimes I_{p} \stackrel{\subset}{\longrightarrow} \Lambda^{-a-1}\left(V_{p-1}\right) \otimes A .
$$

It follows from Lemmas 3.2 and 3.4 that $\zeta^{-a}$ induces an isomorphism in cohomology. Thus $d^{a, b}$ is determined by the inclusion of $\Lambda^{-a-1}\left(V_{p-1}\right) \otimes I_{p}$ into $\Lambda^{-a-1}\left(V_{p-1}\right) \otimes A$.

For $a$ odd, using Lemma 3.4, $\Lambda^{-a-1}\left(V_{p-1}\right)$ is isomorphic to $V_{1}$ plus projectives. Therefore, in this case, $d^{a, b}$ is induced by the inclusion of $I_{p}$ into $A_{p}$. Thus, using Lemma 3.6, if $a$ and $b$ are both odd then $d^{a, b}$ is injective and if $a$ is odd and $b$ is even then $d^{a, b}$ is zero.

For $a$ even, using Lemma $3.4, \Lambda^{-a-1}\left(V_{p-1}\right)$ is isomorphic to $V_{p-1}$ plus projectives. Therefore, in this case, $d^{a, b}$ is induced by the inclusion of $V_{p-1} \otimes I_{p}$ into $V_{p-1} \otimes A_{p} \cong V_{p-1} \otimes V_{p-1}$. By Lemma 3.3. $V_{p-1} \otimes V_{p-1}$ is isomorphic to $V_{1}$ plus projectives. Since $A_{p}$ is isomorphic to $V_{1}$ plus projectives, $V_{p-1} \otimes A_{p}$ is isomorphic to $V_{p-1}$ plus projectives. Thus $d^{a, b}$ is determined by the composition

$$
V_{1} \rightarrow V_{p-1} \otimes I_{p} \rightarrow V_{p-1} \otimes A_{p} \rightarrow V_{p-1}
$$

This map clearly induces the zero map from $H^{1}\left(Q, V_{1}\right)$ to $H^{1}\left(Q, V_{p-1}\right)$. Thus for $a$ even and $b$ odd, $d^{a, b}=0$. To show that $d^{a, b}$ is injective for $a$ even and $b$ even, we need to show that the given map from $V_{1}$ to $V_{p-1}$ is non-zero. It follows from Lemma 3.6, that for the purposes of computing cohomology, the inclusion of $I_{p}$ into $A_{p}$ is the injection of $V_{p-1}$ into $V_{1} \oplus V_{p}$ taking $e^{\prime}$ to $\left(e^{\prime \prime}, \Delta(e)\right)$ where $e, e^{\prime}$ and $e^{\prime \prime}$ denote elements which generate the cyclic $G$-modules $V_{p}, V_{p-1}$ and $V_{1}$ respectively. The cokernel of this map is isomorphic to $V_{2}$. Tensoring over $\mathbf{F}$ is exact so we have a short exact sequence

$$
0 \rightarrow V_{p-1} \otimes V_{p-1} \rightarrow V_{p-1} \otimes\left(V_{1} \oplus V_{p}\right) \rightarrow V_{p-1} \otimes V_{2} \rightarrow 0 .
$$

This gives rise to a long exact sequence in cohomology. Recall that $V_{p-1} \otimes V_{2} \cong$ $V_{p-2} \oplus V_{p}$ (see, for example, [1, Ch.II $\S 7$ Lemma 5]). Thus, modulo projectives, the sequence is $V_{1} \rightarrow V_{p-1} \rightarrow V_{p-2}$. This can only give a long exact sequence on cohomology if the the map from $V_{1}$ to $V_{p-1}$ is non-zero.

Corollary 3.8. For $b>0$ and $a<0$, the spectral sequence satisfies

$$
E_{2}^{a, b}= \begin{cases}\mathbf{F} & \text { if } a=1-p \text { and } b \text { odd } \\ 0 & \text { otherwise. }\end{cases}
$$

It follows from Theorem 3.7 that $\rho^{-1}$ induces an isomorphism from $H^{1}\left(Q, F^{-1}\right)$ to $H^{1}(Q, A)$. This map factors through $H^{1}(Q, I)$ with the first map in the factorisation induced by $\mu$ and the second induced by inclusion. Thus to complete the proof of Theorem 1.2 , it is sufficient to show the following.

Lemma 3.9. The map $\mu$ induces an epimorphism from $H^{1}\left(Q, F^{-1}\right)$ to $H^{1}(Q, I)$. 
Proof. Denote by $\partial^{a, b}$ the connecting homomorphism from $H^{b}\left(Q, K^{a}\right)$ to $H^{b+1}\left(Q, K^{a-1}\right)$ and define a filtration on $H^{1}(Q, I)=H^{1}\left(Q, K^{0}\right)$ by

$$
\mathcal{F}_{t}:=\operatorname{kernel}\left(\partial^{-t, t+1} \circ \partial^{-t+1, t} \circ \cdots \circ \partial^{-2,3} \circ \partial^{-1,2} \circ \partial^{0,1}\right) \text {. }
$$

Since $\partial^{1-p, p}=0$, we have $\mathcal{F}_{p-1}=H^{1}(Q, I)$. Using the long exact sequence in cohomology coming from $0 \rightarrow K^{-1} \rightarrow F^{-1} \rightarrow K^{0} \rightarrow 0$, we see that $\mathcal{F}_{0}$ is the image of $H^{1}\left(Q, F^{-1}\right)$ in $H^{1}(Q, I)$. We will prove the lemma by showing $\mathcal{F}_{0}=\mathcal{F}_{p-1}$.

Using the definition a derived couple (see, for example, [12, Ch. XI, §5]), we have $D_{t+2}^{a-t-1, b+t+1}=\partial^{a-t, b+t} \circ \cdots \circ \partial^{a-1, b+1} \circ \partial^{a, b}\left(D^{a, b}\right)$. If $x \in \mathcal{F}_{t} \backslash \mathcal{F}_{t-1}$ then $\partial^{-t+1, t} \circ \cdots \circ \partial^{0,1}(x)$ is a non-zero element of $D_{t+1}^{-t, t+1}$ which lifts to a non-zero element of $E_{t+1}^{-(t+1), t+1}$. However, it follows from Corollary 3.8 that $E_{2}^{-(t+1), t+1}=0$ for $t \geq 1$. Thus $E_{t+1}^{-(t+1), t+1}=0$ for $t \geq 1$. Therefore $\mathcal{F}_{t}=\mathcal{F}_{0}$ for all $t \geq 1$.

These calculations give the following.

Theorem 3.10. (a) $H^{1}(Q, I)$ is a principal $A^{Q}$-module with generator represented by $z_{p-1}-\left(X_{p}^{p}-X_{1}^{p-1} X_{p}\right)$ and annihilator $\operatorname{Tr}^{Q}(A)$.

(b) The inclusion of I into $A$ induces an $A^{Q}$-module monomorphism of $H^{1}(Q, I)$ to $H^{1}(Q, A)$ taking $\left[z_{p-1}-\left(X_{p}^{p}-X_{1}^{p-1} X_{p}\right)\right]$ to $-\left[N^{Q}\left(X_{p}\right)\right]$.

This completes the proof of Theorem 1.2.

\section{The Noether number of $V_{p+1}$}

In this section we use the description of $\mathbf{F}\left[V_{p+1}\right]^{\mathbf{Z} / p^{2}}$ given in Theorem 1.2 to prove the following.

Theorem 4.1. For $p>2$, the Noether number of $V_{p+1}$ is $p^{2}+p-3$.

Remark 4.2. A Magma [3] calculation shows that for $p=2$, the Noether number of $V_{3}$ is $p^{2}=4$.

For the remainder of this section we will assume that $p \geq 3$. We continue to use the notation described at the beginning of Section 3 , Define $M:=N^{G}\left(x_{p}\right)$ and $N:=N^{G}\left(x_{p+1}\right)$. The theorem is an immediate consequence of the following two lemmas.

Lemma 4.3. The Noether number of $V_{p+1}$ is less than or equal to $p^{2}+p-3$.

Proof. Let $\mathcal{H}$ denote the ideal in $\mathbf{F}\left[V_{p+1}\right]^{L}$ generated by the homogeneous $G$ invariants of positive degree, i.e., $\mathcal{H}=\mathbf{F}\left[V_{p+1}\right]_{+}^{G} \cdot \mathbf{F}\left[V_{p+1}\right]^{L}$. Thus $\mathbf{F}\left[V_{p+1}\right]^{L} / \mathcal{H}$ is a finite dimensional graded algebra, the ring of relative coinvariants. Let $\mathcal{B}$ denote the set of elements of $\mathbf{F}\left[V_{p+1}\right]^{L}$ of the form $\gamma \cdot x_{p}^{j} \cdot N^{L}\left(x_{p+1}\right)^{k}$, with $\gamma$ a monomial in $\left\{x_{1}, \ldots, x_{p-1}\right\}$ of degree at most $p-2$ and $j, k<p$. The methods of Section 3 of [9] show that $\mathcal{B}$ projects to a spanning set in $\mathbf{F}\left[V_{p+1}\right]^{L} / \mathcal{H}$. Therefore $(p-1) p+(p-1)+p-2=p^{2}+p-3$ is an upper bound on the top degree of the relative coinvariants and $\operatorname{Tr}_{L}^{G}(\mathcal{B})$ is a generating set for the ideal $\operatorname{Im}_{\operatorname{Tr}_{L}^{G}}^{G}$. By 
Theorem 1.2, $\mathbf{F}\left[V_{p+1}\right]^{G}$ is generated by $N, M$ and elements from $\operatorname{Im} \operatorname{Tr}_{L}^{G}$. Thus $p^{2}+p-3$ is an upper bound for the Noether number.

Lemma 4.4. The polynomial $\operatorname{Tr}_{L}^{G}\left(\left(N^{L}\left(x_{p+1}\right) x_{p}\right)^{p-1} x_{p-1}^{p-2}\right)$ is indecomposable in $\mathbf{F}\left[V_{p+1}\right]^{G}$. In particular the Noether Number of $V_{p+1}$ is at least $p^{2}+p-3$.

Proof. Define $w:=N^{L}\left(x_{p+1}\right)$ and $z:=\operatorname{Tr}_{L}^{G}\left(w^{p-1} x_{p}^{p-1} x_{p-1}^{p-2}\right)$. Suppose, by way of contradiction, that $z=f_{1} h_{1}+\cdots+f_{s} h_{s}$ where $f_{i}$ and $h_{i}$ are homogeneous positive degree elements of $\mathbf{F}\left[V_{p+1}\right]^{G}$. The degree of $z$ as a polynomial in $x_{p+1}$ is less than $p^{2}$. Thus $N$ does not appear in the decomposition.

We use the graded reverse lexicographic term order with $x_{1}<x_{2}<\cdots<x_{p+1}$ and denote the leading monomial of an element $f \in \mathbf{F}\left[V_{p+1}\right]$ by $\operatorname{LM}(f)$. It is easy to see that $\operatorname{LM}(M)=x_{p}^{p}$. An elementary calculation gives $\operatorname{LM}(z)=x_{p}^{p^{2}-1} x_{p-1}^{p-2}$. By relabelling if necessary, we may assume $\operatorname{LM}\left(f_{i} h_{i}\right) \geq \operatorname{LM}\left(f_{i+1} h_{i+1}\right)$. Thus, either $\operatorname{LM}\left(f_{1} h_{1}\right)=\operatorname{LM}(z)$ or $\operatorname{LM}\left(f_{1} h_{1}\right)=\operatorname{LM}\left(f_{2} h_{2}\right)>\operatorname{LM}(z)$. Without loss of generality, we may assume $f_{1} h_{1}=c M^{m} \alpha$, where $c \in \mathbf{F}$ and $\alpha$ is a (non-constant) product of elements from $\operatorname{Tr}_{L}^{G}(\mathcal{B})$.

Let $\pi$ denote the projection

$$
\pi: \mathbf{F}\left[V_{p+1}\right] \rightarrow \mathbf{F}\left[V_{p+1}\right] /\left(x_{1}, \ldots, x_{p-2}, x_{p-1}^{p-1}\right) \mathbf{F}\left[V_{p+1}\right]
$$

For convenience, write $f \equiv h$ if $\pi(f)=\pi(h)$. Observe that $\pi(z) \neq 0, \pi(w) \equiv x_{p+1}^{p}$ and $\pi(M) \equiv x_{p}^{p}$. Furthermore, the restriction of $\pi$ to $\mathbf{F}\left[V_{p+1}\right]^{L}$ commutes with the action of $Q=G / L$. Thus $\pi \operatorname{Tr}_{L}^{G}(\mathcal{B})=\operatorname{Tr}^{Q} \pi(\mathcal{B})$. If $\beta \in \operatorname{Tr}_{L}^{G}(\mathcal{B})$ with $\pi(\beta) \neq 0$, then $\beta=\operatorname{Tr}_{L}^{G}\left(w^{k} x_{p}^{j} x_{p-1}^{\ell}\right)$ and $\pi(\beta) \equiv x_{p-1}^{\ell} \operatorname{Tr}^{Q}\left(w^{k} x_{p}^{j}\right)$. Summing over the action of $Q$ gives

$$
\begin{aligned}
\operatorname{Tr}^{Q}\left(w^{k} x_{p}^{j}\right) & \equiv \sum_{\lambda \in \mathbf{F}_{p}}\left(x_{p+1}^{p}+\lambda x_{p}^{p}\right)^{k}\left(x_{p}+\lambda x_{p-1}\right)^{j} \\
& \equiv \sum_{\lambda \in \mathbf{F}_{p}}\left(\sum_{t=0}^{k}\left(\begin{array}{l}
k \\
t
\end{array}\right) \lambda^{t} x_{p+1}^{p(k-t)} x_{p}^{t p}\right)\left(\sum_{r=0}^{j}\left(\begin{array}{l}
j \\
r
\end{array}\right) \lambda^{r} x_{p}^{j-r} x_{p-1}^{r}\right) \\
& \equiv \sum_{r=0}^{j} \sum_{t=0}^{k}\left(\sum_{\lambda \in \mathbf{F}_{p}} \lambda^{r+t}\right)\left(\begin{array}{l}
k \\
t
\end{array}\right)\left(\begin{array}{l}
j \\
r
\end{array}\right) x_{p+1}^{p(k-t)} x_{p}^{t p+j-r} x_{p-1}^{r} .
\end{aligned}
$$

Recall that $\sum_{\lambda \in \mathbf{F}_{p}} \lambda^{i}=0$ unless $i$ is a non-zero multiple of $p-1$, in which case the sum is -1 . Therefore $\operatorname{Tr}^{Q}\left(w^{k} x_{p}^{j}\right) \equiv 0$ if $j+k<p-1$. Moreover, if 
$p-1 \leq j+k<2 p-2$, we take $t+r=p-1$ to get

$$
\begin{aligned}
\operatorname{Tr}^{Q}\left(w^{k} x_{p}^{j}\right) & \equiv-\sum_{t=1}^{k}\left(\begin{array}{l}
k \\
t
\end{array}\right)\left(\begin{array}{c}
j \\
p-1-t
\end{array}\right) x_{p+1}^{p(k-t)} x_{p}^{p t+j+t-(p-1)} x_{p-1}^{p-1-t} \\
& \equiv-\sum_{r=p-1-k}^{j}\left(\begin{array}{c}
k \\
p-1-r
\end{array}\right)\left(\begin{array}{l}
j \\
r
\end{array}\right) x_{p+1}^{p(k+r-(p-1))} x_{p}^{p(p-1-r)+j-r} x_{p-1}^{r} \\
& \equiv-\left(\begin{array}{c}
j \\
p-1-k
\end{array}\right) x_{p}^{k(p+1)+j-(p-1)} x_{p-1}^{p-1-k}+x_{p-1}^{p-k} F
\end{aligned}
$$

with $F \in \mathbf{F}\left[x_{p+1}, x_{p}, x_{p-1}\right]$. Since $j \leq p-1$ and $k \leq p-1$, we have $j+k \geq 2 p-2$ only when $j=p-1$ and $k=p-1$. In this case, there is one additional term, $-x_{p}^{p^{2}-p} x_{p-1}^{p-1} \equiv 0$. Since the monomials of degree $k p+j$ taken to zero by $\pi$ are less than $x_{p}^{k p+j-p-2} x_{p-1}^{p-2}$, we have, for $k>0$,

$$
\operatorname{LM}\left(\operatorname{Tr}_{L}^{G}\left(w^{k} x_{p}^{j}\right)\right)=x_{p}^{k(p+1)+j-(p-1)} x_{p-1}^{p-1-k} .
$$

Assume, by way of contradiction, that $\alpha$ is the product of at least two factors, say $\alpha=\beta_{1} \beta_{2} \cdots \beta_{d}$ with $\beta_{i} \in \operatorname{Tr}_{L}^{G}(\mathcal{B})$. Since $\operatorname{LM}\left(c M^{m} \alpha\right) \geq \operatorname{LM}(z)=x_{p}^{p^{2}-1} x_{p-1}^{p-2}$, we have $\operatorname{LM}(\alpha) \geq x_{p}^{p^{2}-m p-1} x_{p-1}^{p-2}$. Therefore, since we are using the graded reverse lexicographic order, $\pi \operatorname{LM}(\alpha) \neq 0$. Furthermore, $\operatorname{LM}\left(\beta_{i}\right)$ divides $\operatorname{LM}(\alpha)$. Thus $\pi\left(\beta_{i}\right) \neq 0$ giving $\beta_{i}=\operatorname{Tr}_{L}^{G}\left(w^{k_{i}} x_{p}^{j_{i}} x_{p-1}^{\ell_{i}}\right)$ with $j_{i}+k_{i} \geq p-1$. Using the formulae above gives

$$
\operatorname{LM}\left(\beta_{1} \beta_{2}\right)=x_{p}^{(p+1)\left(k_{1}+k_{2}\right)+j_{1}+j_{2}-2(p-1)} x_{p-1}^{2(p-1)-k_{1}-k_{2}+\ell_{1}+\ell_{2}} .
$$

Again, using $\operatorname{LM}\left(c M^{m} \alpha\right) \geq \operatorname{LM}(z)$ gives $2(p-1)-k_{1}-k_{2}+\ell_{1}+\ell_{2} \leq p-2$ which simplifies to $k_{1}+k_{2} \geq p+\ell_{1}+\ell_{2} \geq p$. However, $\operatorname{deg}\left(\beta_{1} \beta_{2}\right)=p\left(k_{1}+k_{2}\right)+j_{1}+j_{2}+$ $\ell_{1}+\ell_{2} \leq p^{2}+p-3$, giving $k_{1}+k_{2} \leq p$. Therefore $k_{1}+k_{2}=p$. Furthermore, adding the inequalities $j_{i}+k_{i} \geq p-1$ gives $j_{1}+j_{2}+k_{1}+k_{2}=j_{1}+j_{2}+p \geq 2(p-1)$ which simplifies to $j_{1}+j_{2} \geq p-2$. Thus $\operatorname{deg}\left(\beta_{1} \beta_{2}\right) \geq p\left(k_{1}+k_{2}\right)+j_{1}+j_{2} \geq p^{2}+p-2>$ $\operatorname{deg}\left(c M^{m} \alpha\right)$, giving a contradiction. Thus we must have that $\alpha$ is an element of $\operatorname{Tr}_{L}^{G}(\mathcal{B})$.

It remains to consider the case $f_{1} h_{1}=c M^{m} \alpha$ with $\alpha \in \operatorname{Tr}_{L}^{G}(\mathcal{B})$ and $m>0$. As above, $\pi \operatorname{LM}(\alpha) \neq 0$ gives $\alpha=\operatorname{Tr}_{L}^{G}\left(w^{k} x_{p}^{j} x_{p-1}^{\ell}\right)$ with $k+j \geq p-1$ and $\ell+p-1+k \leq$ $p-2$. The degree constraint gives $p(m+k)+j+\ell=p^{2}+p-3$. Since $\alpha \in \operatorname{Tr}_{L}^{G}(\mathcal{B})$, we have $j, k \leq p-1$ and $\ell \leq p-2$. Thus $j+\ell \leq 2 p-3$. Therefore, either $m+k=p$ and $j+\ell=p-3$ or $m+k=p-1$ and $j+\ell=2 p-3$.

We first consider the case $m+k=p-1$ and $j+\ell=2 p-3$. Since $j \leq p-1$ and $\ell \leq p-2$, we have $j=p-1$ and $\ell=p-2$. Using the above formula for $\pi \operatorname{Tr}^{Q}\left(w^{k} x_{p}^{j}\right)$, with $m>0$,gives

$$
\pi\left(M^{m} \operatorname{Tr}_{L}^{G}\left(w^{p-1-m} x_{p}^{p-1} x_{p-2}^{p-2}\right)\right) \equiv\left(\begin{array}{c}
p-1 \\
m
\end{array}\right) x_{p}^{p^{2}-1-m} x_{p-1}^{p-2+m} \equiv 0 .
$$

Thus LM $\left(M^{m} \operatorname{Tr}_{L}^{G}\left(w^{p-1-m} x_{p}^{p-1} x_{p-2}^{p-2}\right)\right)<\operatorname{LM}(z)$. 
This leaves the case $m+k=p$ and $j+\ell=p-3$. Again using the formula for $\pi \operatorname{Tr}^{Q}\left(w^{k} x_{p}^{j}\right)$ gives

$$
\begin{aligned}
\operatorname{LM}\left(M^{m} \operatorname{Tr}_{L}^{G}\left(w^{p-m} x_{p}^{j} x_{p-1}^{p-3-j}\right)\right) & =x_{p}^{m p+k p+j-(p-1-k)} x_{p-1}^{p-1-k+\ell} \\
& =x_{p}^{p^{2}+j-m+1} x_{p-1}^{p-4+m-j} .
\end{aligned}
$$

However, $j+k \geq p-1$ gives $m-j \leq 1$. Therefore $\operatorname{LM}\left(c M^{m} \alpha\right) \neq \operatorname{LM}(z)$. However, it is possible to choose $\alpha$ and $m$ so that $\operatorname{LM}\left(c M^{m} \alpha\right)=x_{p}^{p^{2}+p-3-s} x_{p-1}^{s}$ for $s=0, \ldots, p-3$. Note that $s=p-4+m-j=\ell+m-1$. Since $m \geq 1, s=0$ occurs only when $m=1$ and $\ell=0$. In general, we may take $m=1,2, \ldots, s+1$ and $\ell=s+1-m$. Define $T_{m, s}:=M^{m} \operatorname{Tr}_{L}^{G}\left(w^{p-m} x_{p}^{p-4+m-s} x_{p-1}^{s+1-m}\right)$. To complete the proof of the lemma, it is sufficient to show that no linear combination of elements of $\mathcal{S}:=\left\{T_{m, s} \mid s=0, \ldots, p-3, m=1,2, \ldots, s+1\right\}$ has lead monomial $\operatorname{LM}(z)=$ $x_{p}^{p^{2}-1} x_{p-1}^{p-2}$. Our argument is essentially Gauss-Jordan elimination applied to $\pi \mathcal{S}$.

Using the above formula for $\pi \operatorname{Tr}^{Q}\left(w^{k} x_{p}^{j}\right)$ gives

$$
T_{m, s} \equiv-\sum_{r=m-1}^{p-4+m-s}\left(\begin{array}{c}
p-m \\
p-1-r
\end{array}\right)\left(\begin{array}{c}
p-4+m-s \\
r
\end{array}\right) x_{p+1}^{p(r-m+1)} x_{p-1}^{r+s+1-m} x_{p}^{*} .
$$

Reindexing with $i=r+s+1-m$ gives

$$
\begin{aligned}
T_{m, s} & \equiv-\sum_{i=s}^{p-2}\left(\begin{array}{c}
p-m \\
p-i+s-m
\end{array}\right)\left(\begin{array}{c}
p-4+m-s \\
i-s+m-1
\end{array}\right) x_{p+1}^{p(i-s)} x_{p-1}^{i} x_{p}^{*} \\
& \equiv-\sum_{i=s}^{p-2}\left(\begin{array}{c}
p-m \\
i-s
\end{array}\right)\left(\begin{array}{c}
p-4+m-s \\
i-s+m-1
\end{array}\right) x_{p+1}^{p(i-s)} x_{p-1}^{i} x_{p}^{*} .
\end{aligned}
$$

Note that in a field of characteristic $p,\left(\begin{array}{c}p-a \\ b\end{array}\right)=(-1)^{a}\left(\begin{array}{c}a-1+b \\ a-1\end{array}\right)$. Thus

$$
T_{m, s} \equiv(-1)^{s+1} \sum_{i=s}^{p-2}\left(\begin{array}{c}
m-1+i-s \\
m-1
\end{array}\right)\left(\begin{array}{c}
i+2 \\
s-m+3
\end{array}\right) x_{p+1}^{p(i-s)} x_{p-1}^{i} x_{p}^{*} .
$$

A simple calculation confirms $\left(\begin{array}{c}a \\ c\end{array}\right)\left(\begin{array}{c}a+b \\ b\end{array}\right)=\left(\begin{array}{c}b+c \\ c\end{array}\right)\left(\begin{array}{c}a+b \\ b+c\end{array}\right)$, giving

$$
\begin{aligned}
T_{m, s} & \equiv(-1)^{s+1} \sum_{i=s}^{p-2}\left(\begin{array}{c}
s+2 \\
m-1
\end{array}\right)\left(\begin{array}{c}
i+2 \\
s+2
\end{array}\right) x_{p+1}^{p(i-s)} x_{p-1}^{i} x_{p}^{p^{2}+p-3-i-p(i-s)} \\
& \equiv(-1)^{s+1}\left(\begin{array}{c}
s+2 \\
m-1
\end{array}\right) \sum_{i=s}^{p-2}\left(\begin{array}{c}
i+2 \\
s+2
\end{array}\right) x_{p+1}^{p(i-s)} x_{p-1}^{i} x_{p}^{p^{2}+p-3-i-p(i-s)}
\end{aligned}
$$

Therefore

$$
(-1)^{s+1} T_{m, s}\left(\begin{array}{c}
s+2 \\
m-1
\end{array}\right)^{-1} \equiv \sum_{i=s}^{p-2}\left(\begin{array}{c}
i+2 \\
s+2
\end{array}\right) x_{p+1}^{p(i-s)} x_{p-1}^{i} x_{p}^{p^{2}+p-3-i-p(i-s)}
$$

is independent of $m$. Thus $\left\{\pi\left(T_{1, s}\right) \mid s=0, \ldots, p-3\right\}$ is a basis for $\pi \mathcal{S}$. Since $\left\{\operatorname{LM}\left(T_{1, s}\right) \mid s=0, \ldots, p-3\right\}=\left\{x_{p}^{p^{2}+p-3-s} x_{p-1}^{s} \mid s=0, \ldots, p-3\right\}$, no linear combination of elements of $\mathcal{S}$ has lead monomial $x_{p}^{p^{2}-1} x_{p-1}^{p-2}$. 
The final assertion of the lemma follows from the fact that deg $z=p^{2}+p-3$.

\section{Decomposing $\mathbf{F}\left[V_{p+1}\right]$}

The main goal of this section is to describe the $\mathbf{F G}$-module decomposition of $\mathbf{F}\left[V_{p+1}\right]$. We do this by considering a basis for $\mathbf{F}\left[V_{p+1}\right]_{d}^{G}$ which is compatible with the length filtration. By Theorem [1.2, $\mathbf{F}\left[V_{p+1}\right]^{G}$ is generated by $M:=N^{G}\left(x_{p}\right)$ and $N:=N^{G}\left(x_{p+1}\right)$ together with elements of the relative transfer, $\operatorname{Tr}_{L}^{G}\left(\mathbf{F}\left[V_{p+1}\right]^{L}\right)$. To identify the summands occurring in the decomposition, we need to determine the lengths of the basis elements.

Suppose $f \in \mathbf{F}\left[V_{p+1}\right]^{G}$. It follows from Lemma 2.5 that if $\ell(f) \geq 2$ then $f \in \sqrt{\operatorname{Im} \operatorname{Tr}_{L}^{G}}=\left(\left(x_{1}, x_{2}, \ldots, x_{p}\right) \mathbf{F}\left[V_{p+1}\right]\right) \cap \mathbf{F}\left[V_{p+1}\right]^{G}$, and if $\ell(f) \geq p+1$ then $f \in \sqrt{\operatorname{Im} \operatorname{Tr}^{G}}=\left(\left(x_{1}\right) \mathbf{F}\left[V_{p+1}\right]\right) \cap \mathbf{F}\left[V_{p+1}\right]^{G}$. Furthermore, since $\operatorname{Tr}_{L}^{G}=\Delta^{p-1}$, if $\ell(f) \geq p$ then $f \in \operatorname{Im}_{L}^{G}$. Since $N=N^{G}\left(x_{p+1}\right)$ has lead term $x_{p+1}^{p^{2}}$, we have that $N \notin\left(x_{1}, x_{2}, \ldots, x_{p}\right) \mathbf{F}\left[V_{p+1}\right]$ and thus $\ell(N)=1$. Similarly since the lead term of $M=N^{G}\left(x_{p}\right)$ is $x_{p}^{p}$, we have that $M \notin\left(x_{1}\right) \mathbf{F}\left[V_{p+1}\right]$ and thus $\ell(M) \leq p$.

Lemma 5.1. Let $1 \leq q<p$. Then

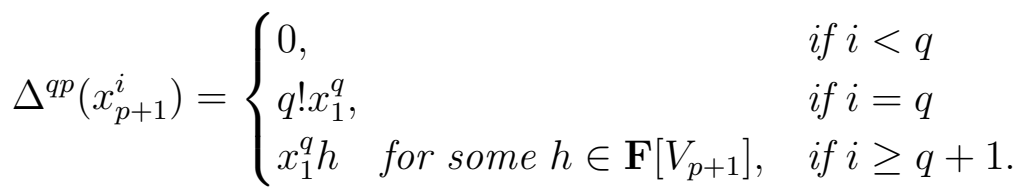

In particular, $\Delta^{q p}\left(x_{p+1}^{i}\right) \in\left(x_{1}^{q}\right) \mathbf{F}\left[V_{p+1}\right]$ for all $i \geq 0$.

Proof. We consider $\Delta^{q p}\left(x_{p+1}^{i}\right)$ using induction on $q$. For $q=1$ we have

$$
\begin{aligned}
\Delta^{p}\left(x_{p+1}^{i}\right) & =\left(x_{p+1}+x_{1}\right)^{i}-x_{p+1}^{i}=\sum_{j=0}^{i-1}\left(\begin{array}{l}
i \\
j
\end{array}\right) x_{1}^{i-j} x_{p+1}^{j} \\
& = \begin{cases}0, & \text { if } i=0 \\
x_{1}, & \text { if } i=1 \\
x_{1} \sum_{j=0}^{i-1}\left(\begin{array}{l}
i \\
j
\end{array}\right) x_{1}^{i-j-1} x_{p+1}^{j}, & \text { if } i \geq 2 .\end{cases}
\end{aligned}
$$

Now take $q+1 \geq 2$. Then

$$
\begin{aligned}
\Delta^{(q+1) p}\left(x_{p+1}^{i}\right) & =\Delta^{q p}\left(\Delta^{p}\left(x_{p+1}^{i}\right)\right) \\
& =\Delta^{q p}\left(\sum_{j=0}^{i-1}\left(\begin{array}{l}
i \\
j
\end{array}\right) x_{1}^{i-j} x_{p+1}^{j}\right) \\
& =\sum_{j=0}^{i-1}\left(\begin{array}{l}
i \\
j
\end{array}\right) x_{1}^{i-j} \Delta^{q p}\left(x_{p+1}^{j}\right) .
\end{aligned}
$$

\footnotetext{
${ }^{1}$ Use any monomial order with $x_{1}<x_{2}<\cdots<x_{p+1}$.
} 
By induction this gives

$$
\begin{aligned}
\Delta^{(q+1) p}\left(x_{p+1}^{i}\right) & =\sum_{j=q}^{i-1}\left(\begin{array}{l}
i \\
j
\end{array}\right) x_{1}^{i-j} \Delta^{q p}\left(x_{p+1}^{j}\right) \\
& =\left\{\begin{array}{lr}
0, & \text { if } i-1<q \\
\left(\begin{array}{c}
q+1 \\
q
\end{array}\right) x_{1} \Delta^{q p}\left(x_{p+1}^{q}\right), & \text { if } i-1=q \\
x_{1} \sum_{j=q}^{i-1}\left(\begin{array}{l}
i \\
j
\end{array}\right) x_{1}^{i-j-1} \Delta^{q p}\left(x_{p+1}^{j}\right), & \text { if } i-1>q
\end{array}\right. \\
& =\left\{\begin{array}{lr}
0, & \text { if } i<q+1 \\
(q+1) x_{1} q ! x_{1}^{q}, & \text { if } i=q+1 \\
x_{1} \sum_{j=q}^{i-1} x_{1}^{q} h_{j} & \text { where } h_{j} \in \mathbf{F}\left[V_{p+1}\right], \quad \text { if } i \geq q+1
\end{array}\right. \\
& =\left\{\begin{array}{lr}
0, & \text { if } i<q+1 \\
(q+1) ! x_{1}^{q+1}, & \text { if } i \geq q+1 \\
x_{1}^{q+1} h \quad \text { for some } h \in \mathbf{F}\left[V_{p+1}\right], & \text { if } i .
\end{array}\right.
\end{aligned}
$$

Proposition 5.2. Let $f$ be a non-zero element of $\mathbf{F}\left[V_{p+1}\right]^{G}$ and let $1 \leq q<p$. Then $\ell(f) \geq q p+1 \Longleftrightarrow f \in \operatorname{Im} \Delta^{q p} \Longleftrightarrow f \in\left(x_{1}^{q}\right) \mathbf{F}\left[V_{p+1}\right]^{G}$.

Proof. The first equivalence is just the definition of length. For the second equivalence, first suppose that $f=\Delta^{q p}(F) \in \operatorname{Im} \Delta^{q p}$. Write $F=\sum_{i=0}^{r} f_{i} x_{p+1}^{i}$ where each $f_{i} \in \mathbf{F}\left[x_{1}, x_{2}, \ldots, x_{p}\right]$. Then $f=\Delta^{q p}(F)=\sum_{i=0}^{r} f_{i} \Delta^{q p}\left(x_{p+1}^{i}\right) \in\left(x_{1}^{q}\right) \mathbf{F}\left[V_{p+1}\right]^{G}$ by the previous lemma. Conversely, suppose that $f \in\left(x_{1}^{q}\right) \mathbf{F}\left[V_{p+1}\right]^{G}$ and write $f=x_{1}^{q} f^{\prime}$ where $f^{\prime} \in \mathbf{F}\left[V_{p+1}\right]^{G}$. Then

$$
\Delta^{q p}\left(\frac{x_{p+1}^{q} f^{\prime}}{q !}\right)=\frac{f^{\prime}}{q !} \Delta^{q p}\left(x_{p+1}^{q}\right)=\frac{f^{\prime}}{q !} q ! x_{1}^{q}=f .
$$

Proposition 5.3. Let $f$ be a non-zero element of $\mathbf{F}\left[V_{p+1}\right]^{G}$ and write $f=x_{1}^{q} f^{\prime}$ where $x_{1}$ does not divide $f^{\prime}$. If $q \geq p$ then $\ell(f)=p^{2}$. Otherwise $\ell(f)=q p+\ell\left(f^{\prime}\right)$.

Proof. Applying Lemma 5.1 gives $\Delta^{(p-1) p}\left(x_{p+1}^{p-1}\right)=(p-1) ! x_{1}^{p-1}=-x_{1}^{p}$. Furthermore, $\Delta^{p}\left(x_{p}\right)=0$. Thus

$$
\begin{aligned}
\Delta^{p^{2}-1}\left(x_{p+1}^{p-1} x_{p}\right) & =\Delta^{p-1}\left(\left(\Delta^{p}\right)^{p-1}\left(x_{p+1}^{p-1} x_{p}\right)\right)=\Delta^{p-1}\left(x_{p} \Delta^{(p-1) p}\left(x_{p+1}^{p-1}\right)\right) \\
& =-\Delta^{p-1}\left(x_{p} x_{1}^{p-1}\right)=-x_{1}^{p-1} \Delta^{p-1}\left(x_{p}\right)=-x_{1}^{p} .
\end{aligned}
$$

Therefore $\Delta^{p^{2}-1}\left(-x_{p+1}^{p-1} x_{p} f^{\prime}\right)=x_{1}^{p} f^{\prime}$. This implies that if $q \geq p$ then $\ell(f)=p^{2}$. Suppose then that $q<p$. By Proposition 5.2, we have $q p \leq \ell(f)-1$. Since $x_{1}^{q+1}$ does not divide $f$, Proposition 5.2 also implies that $\ell(f)-1<(q+1) p$. Write $\ell(f)-1=q p+r$ where $0 \leq r \leq p-1$ and define $s:=\ell\left(f^{\prime}\right)-1$. Since $x_{1}$ does not divide $f^{\prime}$, Lemma 2.5 implies that $0 \leq s \leq p-1$. We will show that $r=s$. 
Clearly there exists $F \in \mathbf{F}\left[V_{p+1}\right]$ such that $f=\Delta^{q p+r}(F)$. Therefore $f=$ $\Delta^{r}\left(\Delta^{q p}(F)\right)=\Delta^{r}\left(x_{1}^{q} F^{\prime}\right)$ for some $F^{\prime} \in \mathbf{F}\left[V_{p+1}\right]$. Hence $x_{1}^{q} f^{\prime}=f=x_{1}^{q} \Delta^{r}\left(F^{\prime}\right)$ and therefore $f^{\prime}=\Delta^{r}\left(F^{\prime}\right)$. Hence $s+1=\ell\left(f^{\prime}\right) \geq r+1$.

Conversely we may write $f^{\prime}=\Delta^{s}\left(F^{\prime \prime}\right)$ for some $F^{\prime \prime} \in \mathbf{F}\left[V_{p+1}\right]$. Since $s \leq$ $p-1$ we have $\Delta^{p}\left(F^{\prime \prime}\right)=\Delta^{p-s}\left(\Delta^{s}\left(F^{\prime \prime}\right)\right)=\Delta^{p-s}\left(f^{\prime}\right)=0$. This shows that $F^{\prime \prime} \in \mathbf{F}\left[V_{p+1}\right]^{L}$. Thus $\Delta^{q p+s}\left(x_{p+1}^{q} F^{\prime \prime}\right)=\left(\Delta^{s}\left(\Delta^{p}\right)^{q}\right)\left(x_{p+1}^{q} F^{\prime \prime}\right)=\Delta^{s}\left(q ! x_{1}^{q} F^{\prime \prime}\right)=$ $q ! x_{1}^{q} \Delta^{s}\left(F^{\prime \prime}\right)=q ! x_{1}^{q} f^{\prime}=q ! f$ where $q ! \neq 0$ since $q<p$. This shows that $f \in$ $\operatorname{Im} \Delta^{q p+s}$ and thus $q p+r+1=\ell(f) \geq q p+s+1$. Therefore $r=s$ as required.

Proposition 5.4. Let $f$ be a non-zero element in the image of the relative transfer, $\operatorname{Tr}_{L}^{G}\left(\mathbf{F}\left[V_{p+1}\right]^{L}\right)$. Suppose that $x_{1}$ does not divide $f$. Then $\ell(f)=p$.

Proof. Since $x_{1}$ does not divide $f$, Lemma 2.5 implies that $\ell(f) \leq p$. Conversely $\operatorname{Tr}_{L}^{G}=1+\sigma+\sigma^{2}+\cdots+\sigma^{p-1}=\Delta^{p-1}$. Thus the hypothesis that $f \in \operatorname{Im} \operatorname{Tr}_{L}^{G}$ implies that $\ell(f) \geq p$.

Remark 5.5. Since elements in $\operatorname{Tr}^{G}\left(\mathbf{F}\left[V_{p+1}\right]\right)$ have length $p^{2}$, it is clear that

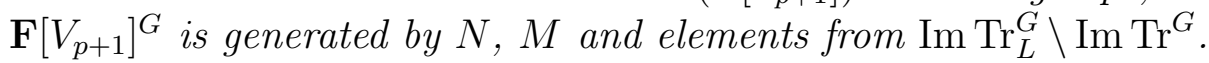

Proposition 5.6. $\ell\left(M^{j}\right)=j+1$ for all $j=0,1, \ldots, p-1$. In particular $M^{j}$ lies in the image of the relative transfer, $\operatorname{Tr}_{L}^{G}\left(\mathbf{F}\left[V_{p+1}\right]^{L}\right)$, if and only if $j \geq p-1$.

Proof. From Theorem [1.2, $\mathbf{F}\left[V_{p+1}\right]^{G}$ is generated by $M, N$ and elements from $\operatorname{Im} \operatorname{Tr}_{L}^{G}$. Note that $\operatorname{deg}(M)=p$ and $\operatorname{deg}(N)=p^{2}$. Thus for $d<p^{2}$, if $p$ does not divide $d$, we have $\mathbf{F}\left[V_{p+1}\right]_{d}^{G}=\operatorname{Tr}_{L}^{G}\left(\mathbf{F}\left[V_{p+1}\right]_{d}^{L}\right)$ and, if $d=i p$ with $i<p$, $\mathbf{F}\left[V_{p+1}\right]_{d}^{G}=\mathbf{F} \cdot M^{i}+\operatorname{Tr}_{L}^{G}\left(\mathbf{F}\left[V_{p+1}\right]_{i p}^{L}\right)$. Fix $j \in\{1,2, \ldots, p-1\}$. Choose a basis, $\mathcal{B}$, for $\mathbf{F}\left[V_{p+1}\right]_{j p}^{G}$ so that $\mathcal{B}$ is compatible with the length filtration. Applying Proposition 2.3 gives a decomposition

$$
\mathbf{F}\left[V_{p+1}\right]_{j p}=\bigoplus_{\alpha \in \mathcal{B}} V(\alpha)
$$

Suppose $f \in \mathcal{L}_{p}^{G}\left(\mathbf{F}\left[V_{p+1}\right]\right) \cap \mathcal{B}$. Then $f \in \operatorname{Tr}_{L}^{G}\left(\mathbf{F}\left[V_{p+1}\right]^{L}\right)$. If $x_{1}$ does not divide $f$, then by Proposition 5.4 $\ell(f)=p$. Suppose $x_{1}$ does divide $f$. Write $f=x_{1}^{q} f^{\prime}$ where $x_{1}$ does not divide $f^{\prime}$. If $f^{\prime} \in \operatorname{Tr}_{L}^{G}\left(\mathbf{F}\left[V_{p+1}\right]^{L}\right)$, then by Proposition 5.3 and Proposition 5.4, $\ell(f)$ is a multiple of $p$. If $f^{\prime} \notin \operatorname{Tr}_{L}^{G}\left(\mathbf{F}\left[V_{p+1}\right]^{L}\right)$, then $f^{\prime}=c M^{i}+f^{\prime \prime}$ for some non-zero $c \in \mathbf{F}$ and some $f^{\prime \prime} \in \operatorname{Tr}_{L}^{G}\left(\mathbf{F}\left[V_{p+1}\right]^{L}\right)$. Thus $\operatorname{deg}\left(f^{\prime}\right)=i p$ and $q=(j-i) p$. Thus $q>p$ and by Proposition [5.3, $\ell(f)$ is $p^{2}$. Hence, for any $f \in \mathcal{L}_{p}^{G}\left(\mathbf{F}\left[V_{p+1}\right]\right) \cap \mathcal{B}, \ell(f)$ is a multiple of $p$. Therefore $p$ divides the dimension of

$$
\bigoplus_{\alpha \in \mathcal{L}_{p}^{G}\left(\mathbf{F}\left[V_{p+1}\right]\right) \cap \mathcal{B}} V(\alpha) .
$$

Since $\operatorname{dim} \mathbf{F}\left[V_{p+1}\right]_{p j}=\left(\begin{array}{c}p+p j \\ p j\end{array}\right)$, Lucas' Lemma (see, for example, [7]) implies that $\operatorname{dim} \mathbf{F}\left[V_{p+1}\right]_{p j} \equiv\left(\begin{array}{c}j+1 \\ j\end{array}\right)\left(\begin{array}{l}0 \\ 0\end{array}\right)(\bmod p)$. Thus $\operatorname{dim} \mathbf{F}\left[V_{p+1}\right]_{p j} \equiv j+1(\bmod p)$. This shows that $M^{j} \notin \operatorname{Tr}_{L}^{G}\left(\mathbf{F}\left[V_{p+1}\right]^{L}\right)$ for all $j \leq p-2$ and that $M^{p-1} \in \operatorname{Tr}_{L}^{G}\left(\mathbf{F}\left[V_{p+1}\right]^{L}\right)$. Furthermore, for $1 \leq j<p-1$ we have $\ell\left(M^{j}\right)<p$ and $\ell\left(M^{j}\right) \equiv j+1(\bmod p)$ and thus $\ell\left(M^{j}\right)=j+1$. Since $\mathbf{F}\left[V_{p+1}\right]_{0} \cong \mathbf{F}$, it is clear that $\ell\left(M^{0}\right)=1$. 
Define $\mathbf{F}\left[V_{p+1}\right]^{b}$ to be the span of the monomials in $\mathbf{F}\left[V_{p+1}\right]$ which, as polynomials in $x_{p+1}$, have degree less than $p^{2}$. It follows from the proof of Proposition 2.6 that, as $\mathbf{F} G$-modules, $\mathbf{F}\left[V_{p+1}\right]=N \mathbf{F}\left[V_{p+1}\right] \oplus \mathbf{F}\left[V_{p+1}\right]^{b}$. Thus a decomposition of $\mathbf{F}\left[V_{p+1}\right]^{b}$ gives a decomposition of $\mathbf{F}\left[V_{p+1}\right]$. Therefore the following theorem implies Theorem 1.3 .

Theorem 5.7. (i) For $d<p^{2}-p$, an $\mathbf{F} G$-module decomposition of $\mathbf{F}\left[V_{p+1}\right]_{d}^{b}$ includes precisely one non-induced indecomposable summand. Divide $p$ into $d$ to get $d=b p+c$ with $0 \leq c<p$. The non-induced summand is isomorphic to $V_{c p+b+1}$ and the decomposition may be chosen so that the socle of the non-induced indecomposable is spanned by $x_{1}^{c} M^{b}$.

(ii) For $d \geq p^{2}-p, \mathbf{F}\left[V_{p+1}\right]_{d}^{b}$ is a direct sum of indecomposable induced modules.

Proof. Fix $d$ and choose a basis, $\mathcal{B}$, for $\left(\mathbf{F}\left[V_{p+1}\right]_{d}^{b}\right)^{G}$, so that $\mathcal{B}$ is compatible with the length filtration. Applying Proposition 2.3 gives a decomposition

$$
\mathbf{F}\left[V_{p+1}\right]_{d}^{b}=\bigoplus_{\alpha \in \mathcal{B}} V(\alpha)
$$

with $V(\alpha) \cong V_{\ell(\alpha)}$. Write $\alpha=x_{1}^{i} \alpha^{\prime}$ where $x_{1}$ does not divide $\alpha^{\prime}$. If $i \geq p$, then by Proposition 5.3, $\ell(\alpha)=p^{2}$ and $V(\alpha)$ is projective. Suppose $i<p$. If $\alpha^{\prime} \in \operatorname{Im} \operatorname{Tr}_{L}^{G}$, then by Proposition 5.4, $\ell\left(\alpha^{\prime}\right)=p$. Thus, using Proposition 5.3, $\ell(\alpha)=i p+p$ and $V(\alpha)$ is an induced module. Suppose $\alpha^{\prime} \notin \operatorname{Im} \operatorname{Tr}_{L}^{G}$. Then $\alpha^{\prime}=k M^{j}+h$ where $j<p-1, k$ is a non-zero element of $\mathbf{F}$ and $h \in \operatorname{Im} \operatorname{Tr}_{L}^{G}$. It follows from Proposition 5.6 that $\ell\left(\alpha^{\prime}\right)=j+1$. Applying Proposition 5.3 gives $\ell(\alpha)=p i+j+1$. This last case is the only way in which a non-induced summand can appear in the decomposition. Note that in this case, $d=p j+i$ with $0 \leq i<p$ and $j<p-1$, giving $d \leq(p-2) p+(p-1)=p^{2}-p-1, i=c$ and $j=b$. Suppose, by way of contradiction, that $\alpha_{1}, \alpha_{2} \in \mathcal{B}$ are distinct elements both having length $c p+b+1$. Then $\alpha_{1}=x_{1}^{c}\left(k_{1} M^{b}+h_{1}\right)$ and $\alpha_{2}=x_{1}^{c}\left(k_{2} M^{b}+h_{2}\right)$ with $k_{i} \in \mathbf{F} \backslash\{0\}$ and $h_{i} \in \operatorname{Im~}_{\operatorname{Tr}_{L}^{G}}^{G}$. From Proposition 5.3, $\ell\left(k_{2} \alpha_{1}-k_{1} \alpha_{2}\right) \geq c p+p>c p+b+1$ contradicting the fact that $\mathcal{B}$ is compatible with the length filtration.

Remark 5.8. The strong form of the Hughes-Kemper Periodicity Conjecture [10, Conjecture 4.6] states that for $p^{m-1}<n \leq p^{m}$ and $d>p^{m}-n, \mathbf{F}\left[V_{n}\right]_{d}^{b}$ is induced. The preceding Theorem verifies the conjecture for $n=p+1$.

\section{Appendix}

In this appendix we will derive generating functions which give the multiplicities of the indecomposable $\mathbf{F} G$-modules as summands in $\mathbf{F}\left[V_{p+1}\right]_{n}$. We also derive the Hilbert series for $\mathbf{F}\left[V_{p+1}\right]^{G}$.

Throughout the appendix we will write $n=\alpha p^{2}+\beta p+\gamma$ where $0 \leq \beta, \gamma \leq p-1$. If $\beta \neq p-1$ then by Theorem 1.3 we know that $\mathbf{F}\left[V_{p+1}\right]_{n}$ contains exactly one non-induced summand, $V_{d(n)}$ where $d(n)=\gamma p+\beta+1$. For convenience we will also define $d(n)=\gamma p+\beta+1=(\gamma+1) p$ when $\beta=p-1$. 
Define integer valued functions $a_{1}(n), a_{2}(n), \ldots, a_{p}(n)$ by

$$
\mathbf{F}\left[V_{p+1}\right]_{n} \cong V_{d(n)} \oplus a_{1}(n) V_{p} \oplus a_{2}(n) V_{2 p} \oplus \cdots \oplus a_{p}(n) V_{p^{2}}
$$

By Propositions 5.2 and 5.3, an invariant $f$ spans the socle of a copy of $V_{i p}$ where $p>i \geq 2$, if and only if $f=x_{1} h$ where the invariant $h$ spans the socle of a copy of $V_{(i-1) p}$. Clearly if $n=\operatorname{deg}(f)$ then $\operatorname{deg}(h)=n-1 \geq 0$. This means that for all $2 \leq i \leq p-1$ we have

$$
a_{i}(n)= \begin{cases}0, & \text { if } n=0,1 \\ a_{i-1}(n-1), & \text { if } n \geq 1\end{cases}
$$

Similarly, Proposition 5.3 with $q=p-1$ and $q=p$ combined with Theorem 1.3 implies that

$$
a_{p}(n)= \begin{cases}0, & \text { if } n=0,1 \\ a_{p}(n-1)+a_{p-1}(n-1), & \text { if } p \text { does not divide } n \\ a_{p}(n-1)+a_{p-1}(n-1)+1, & \text { if } p \text { divides } n \text { and } n \neq 0\end{cases}
$$

Furthermore comparing dimensions in the decomposition (6.1) yields the equation:

$$
\left(\begin{array}{c}
n+p \\
p
\end{array}\right)=d(n)+p a_{1}(n)+2 p a_{2}(n)+\cdots+p^{2} a_{p}(n) .
$$

Introduce the generating functions:

$$
\begin{aligned}
& D(x)=\sum_{n=0}^{\infty} d(n) x^{n} \\
& A_{i}(x)=\sum_{n=0}^{\infty} a_{i}(n) x^{n} \text { for } i=1,2, \ldots, p .
\end{aligned}
$$

In terms of these generating functions, the above recursive conditions, (6.2) and (6.3), become:

$$
\begin{aligned}
A_{i}(x) & =\sum_{n=0}^{\infty} a_{i}(n) x^{n} \\
& =a_{i}(0)+x \sum_{n=1}^{\infty} a_{i-1}(n-1) x^{n-1} \\
& =x A_{i-1} \quad(\text { for } i=2,3, \ldots, p-1)
\end{aligned}
$$


and

$$
\begin{aligned}
& A_{p}(x)= \sum_{n=0}^{\infty} a_{p}(n) x^{n} \\
&= a_{p}(0)+x \sum_{n=1}^{\infty}\left(a_{p}(n-1)+a_{p-1}(n-1)+\delta_{n}^{0}\right) x^{n-1} \\
& \text { where } \delta_{n}^{0}= \begin{cases}1, & \text { if } n \equiv 0 \quad(\bmod p) ; \\
0, & \text { otherwise }\end{cases} \\
&=x A_{p}(x)+x A_{p-1}(x)+\sum_{n=1}^{\infty} x^{n p} \\
&=x A_{p}(x)+x A_{p-1}(x)+\frac{x^{p}}{1-x^{p}}
\end{aligned}
$$

Again using the generating functions, the dimension equation (6.4) becomes

$$
\frac{1}{(1-x)^{p+1}}=D(x)+p A_{1}(x)+2 p A_{2}(x)+\cdots+p^{2} A_{p}(x) .
$$

Substituting $A_{2}(x)=x A_{1}(x), A_{3}(x)=x^{2} A_{1}(x), \ldots, A_{p-1}(x)=x^{p-2} A_{1}(x)$, we are left with the following two equations in $A_{1}$ and $A_{p}$ :

$$
\begin{gathered}
A_{p}(x)=x A_{p}(x)+x^{p-1} A_{1}(x)+\frac{x^{p}}{1-x^{p}} \\
p A_{1}(x)+2 p x A_{1}(x)+\ldots+\left(p^{2}-p\right) x^{p-2} A_{1}(x)+p^{2} A_{p}(x)+D(x)=\frac{1}{(1-x)^{p+1}} .
\end{gathered}
$$

Collecting terms this system becomes:

$$
\begin{aligned}
-x^{p-1} A_{1}(x)+(1-x) A_{p}(x) & =\frac{x^{p}}{1-x^{p}} \\
p\left(1+2 x+3 x^{2}+\cdots+(p-1) x^{p-2}\right) A_{1}(x)+p^{2} A_{p}(x) & =-D(x)+\frac{1}{(1-x)^{p+1}} .
\end{aligned}
$$

Note that, as is easily verified by integration, we have

$$
1+2 x+3 x^{2}+\cdots+m x^{m-1}=\frac{1-(m+1) x^{m}+m x^{m+1}}{(1-x)^{2}} .
$$

Thus the above system of equations becomes

$$
\begin{aligned}
-x^{p-1} A_{1}(x)+(1-x) A_{p}(x) & =\frac{x^{p}}{1-x^{p}} \\
p\left(\frac{1-p x^{p-1}+(p-1) x^{p}}{(1-x)^{2}}\right) A_{1}(x)+p^{2} A_{p}(x) & =-D(x)+\frac{1}{(1-x)^{p+1}} .
\end{aligned}
$$


Solving for $A_{1}$ and $A_{p}$ yields

$$
\begin{gathered}
A_{1}(x)=\left(\frac{-p^{2} x^{p}}{1-x^{p}}+\frac{1}{(1-x)^{p}}-(1-x) D(x)\right)\left(\frac{1-x}{p\left(1-x^{p}\right)}\right) \\
A_{p}(x)=\left(\frac{1-p x^{p-1}+(p-1) x^{p}}{(1-x)^{2}}\left(\frac{p x^{p}}{1-x^{p}}\right)+\frac{x^{p-1}}{(1-x)^{p+1}}-x^{p-1} D(x)\right)\left(\frac{1-x}{p\left(1-x^{p}\right)}\right) .
\end{gathered}
$$

Thus a closed form for $D(x)$ will yield closed forms for $A_{1}(x)$ and $A_{p}(x)$. To obtain a closed expression for $D(x)$ we observe that the sequence $\{d(n)\}_{n=0}^{\infty}$ is the sum of two periodic sequences, one of period $p$ and one of period $p^{2}$. From this using Equation (6.6) twice we get

$$
\begin{aligned}
D(x)= & \left(\sum_{\gamma=0}^{p-1} p \gamma x^{\gamma}\right) \frac{1}{1-x^{p}}+\left(\sum_{\beta=0}^{p-1} \sum_{\gamma=0}^{p-1}(\beta+1) x^{p \beta+\gamma}\right) \frac{1}{1-x^{p^{2}}} \\
= & p x\left(\sum_{\gamma=0}^{p-1} \gamma x^{\gamma-1}\right) \frac{1}{1-x^{p}}+\left(\sum_{\beta=0}^{p-1}(\beta+1) x^{p \beta}\right)\left(\sum_{\gamma=0}^{p-1} x^{\gamma}\right) \frac{1}{1-x^{p^{2}}} \\
= & p x\left(\frac{1-p x^{p-1}+(p-1) x^{p}}{(1-x)^{2}}\right) \frac{1}{1-x^{p}} \\
& +\left(\frac{1-(p+1)\left(x^{p}\right)^{p}+p\left(x^{p}\right)^{p+1}}{\left(1-x^{p}\right)^{2}}\right)\left(\frac{1-x^{p}}{1-x}\right) \frac{1}{1-x^{p^{2}}} .
\end{aligned}
$$

Substituting this expression into the expression for $A_{p}$ given above and simplifying yields the following:

$$
A_{p}(x)=\frac{1}{p\left(1-x^{p}\right)}\left(\frac{x^{p-1}}{(1-x)^{p}}-\frac{x^{p-1}-(p+1) x^{p^{2}+p-1}+p x^{p^{2}+2 p-1}}{\left(1-x^{p}\right)\left(1-x^{p^{2}}\right)}\right) .
$$

Using this expression for $A_{p}(x)$ in (6.7) gives

$$
A_{1}(x)=\frac{-x}{1-x^{p}}+\frac{1-x}{p\left(1-x^{p}\right)}\left(\frac{1}{(1-x)^{p}}-\frac{1-(p+1) x^{p^{2}}+p x^{p^{2}+p}}{\left(1-x^{p}\right)\left(1-x^{p^{2}}\right)}\right) .
$$

In the above description, the summand $V_{d(n)}$ is sometimes an induced summand. More precisely, this happens exactly when $\beta=p-1$. Thus if we decompose the induced component

$$
\left(\mathbf{F}\left[V_{p+1}\right]_{n}\right)_{\text {induced }} \cong b_{1}(n) V_{p} \oplus b_{2}(n) V_{2 p} \oplus \cdots \oplus b_{p}(n) V_{p^{2}}
$$

we have

$$
b_{i}(n)= \begin{cases}a_{i}(n)+1, & \text { if } \gamma=i-1 \text { and } \beta=p-1 \\ a_{i}(n), & \text { otherwise }\end{cases}
$$

Thus the generating function $B_{i}(x)=\sum_{n=0}^{\infty} b_{i}(n) x^{n}$ is given by

$$
B_{i}(x)=A_{i}(x)+\frac{x^{p^{2}-p+i-1}}{1-x^{p^{2}}}=x^{i-1} A_{1}(x)+\frac{x^{p^{2}-p+i-1}}{1-x^{p^{2}}} .
$$


Note that the Hilbert series of the ring of invariants $\mathbf{F}\left[V_{p+1}\right]^{\mathbf{Z} / p^{2}}$ is given by $\mathcal{H}\left(\mathbf{F}\left[V_{p+1}\right]^{\mathbf{Z} / p^{2}}, x\right)=\frac{1}{1-x}+\sum_{i=1}^{p} A_{i}(x)$. Repeatedly using the recursive equation for $A_{p}(n)(6.5)$ we obtain

$$
\begin{aligned}
A_{p} & =x A_{p}+x A_{p-1}+\frac{x^{p}}{1-x^{p}} \\
& =x\left(x A_{p}+x A_{p-1}+\frac{x^{p}}{1-x^{p}}\right)+x A_{p-1}+\frac{x^{p}}{1-x^{p}} \\
& =x^{2} A_{p}+\left(x^{2}+x\right) A_{p-1}+(x+1) \frac{x^{p}}{1-x^{p}} \\
& =x^{2}\left(x A_{p}+x A_{p-1}+\frac{x^{p}}{1-x^{p}}\right)+\left(x^{2}+x\right) A_{p-1}+(x+1) \frac{x^{p}}{1-x^{p}} \\
& =x^{3} A_{p}+\left(x^{3}+x^{2}+x\right) A_{p-1}+\left(x^{2}+x+1\right) \frac{x^{p}}{1-x^{p}} \\
& =x^{p-1} A_{p}+\left(x^{p-1}+x^{p-2}+\cdots+x\right) A_{p-1}+\left(x^{p-2}+x^{p-3}+\cdots+1\right) \frac{x^{p}}{1-x^{p}} \\
& =x^{p-1}\left(A_{p}+\left(1+x^{-1}+\cdots+x^{-(p-2)}\right) A_{p-1}\right)+\left(\frac{1-x^{p-1}}{1-x}\right) \frac{x^{p}}{1-x^{p}} \\
& =x^{p-1}\left(A_{p}+\left(A_{p-1}+A_{p-2}+\cdots+A_{1}\right)+\left(\frac{1}{1-x}-\frac{1}{1-x^{p}}\right)\right) \\
& =x^{p-1}\left(\mathcal{H}\left(\mathbf{F}\left[V_{p+1}\right]^{\mathbf{Z} / p^{2}}, x\right)-\frac{1}{1-x^{p}}\right) \cdot
\end{aligned}
$$

Therefore

$$
\begin{aligned}
\mathcal{H}\left(\mathbf{F}\left[V_{p+1}\right]^{\mathbf{Z} / p^{2}}, x\right) & =\frac{1}{x^{p-1}} A_{p}(x)+\frac{1}{1-x^{p}} \\
& =\frac{1}{p\left(1-x^{p}\right)}\left(\frac{1}{(1-x)^{p}}+\frac{(p-1)-p x^{p}+x^{p^{2}}}{\left(1-x^{p}\right)\left(1-x^{p^{2}}\right)}\right) .
\end{aligned}
$$

\section{REFERENCES}

[1] J.L. Alperin, Local representation theory, Cambridge Univ. Press, 1986.

[2] D.J. Benson, Representations and cohomology I: Basic representation theory of finite groups and associative algebras, Cambridge Univ. Press, 1991.

[3] W. Bosma, J.J. Cannon and C. Playoust, The Magma algebra system I: the user language, J. Symbolic Comput. 24 (1997) 235-265.

[4] W. Bruns and J. Herzog, Cohen-Macaulay rings, Cambridge Univ. Press, 1993.

[5] H.E.A. Campbell, I.P. Hughes, G. Kemper, R.J. Shank and D.L. Wehlau, Depth of modular invariant rings, Transform. Groups 5 (2000) no. 1, 21-34.

[6] L. Evens, The Cohomology of Groups, Oxford Univ. Press, 1991.

[7] N.J. Fine, Binomial coefficients modulo a prime, Amer. Math. Monthly 54 (1947) 589-592. 
[8] P. Fleischmann, Relative Trace Ideals and Cohen-Macaulay Quotients of Modular Invariant Rings, in: P. Dräxler, G.O. Michler, C. M. Ringel, eds., Computational Methods for Representations of Groups and Algebras, Euroconference in Essen, April 1-5 1997, Progress in Mathematics 173, Birkhäuser, 1999.

[9] P. Fleischmann, M. Sezer, R.J. Shank and C.F. Woodcock, The Noether numbers for cyclic groups of prime order, Advances in Mathematics 207 (2006) no. 1, 149-155.

[10] I. Hughes and G. Kemper, Symmetric powers of modular representations, Hilbert series and degree bounds, Comm. in Alg. 28 (2000) 2059-2088.

[11] D.B. Karagueuzian and P. Symonds, The module structure of a group action on a polynomial ring: a finiteness theorem, J. Amer. Math. Soc., PII: S 0894-0347(07)00563-2

[12] S. Mac Lane, Homology, Springer-Verlag, 1963.

[13] R.J. Shank, S.A.G.B.I. bases for rings of formal modular seminvariants, Comment. Math. Helv. 73 (1998) no. 4, 548-565.

[14] R.J. Shank and D.L. Wehlau, Noether numbers for subrepresentations of cyclic groups of prime order, Bull. London Math. Soc. 34 (2002) 438-450.

[15] R.J. Shank and D.L. Wehlau, Computing modular invariants of p-groups, J. Symb. Comp. 34 (2002) no. 5, 307-327.

[16] P. Symonds, Cyclic group actions on polynomial rings, Bull. London Math. Soc., doi: $10.1112 / \mathrm{blms} / \mathrm{bdl} 023$

Institute of Mathematics, Statistics \& Actuarial Science, University of Kent, CANTERbury, CT2 7NF, UK

E-mail address: R.J.Shank@kent.ac.uk

Department of Mathematics \& Computer Science, Royal Military College, Kingston, Ontario, Canada, K7K 7B4

E-mail address: wehlau@rmc.ca 